Cunren Liang

\title{
InSAR Time Series Analysis of L-band Wide- Swath SAR Data Acquired by ALOS-2
}

Jet Propulsion Laboratory, California Institute of Technology, Pasadena, CA, USA

cunrenl@caltech.edu

Zhen Liu

Jet Propulsion Laboratory, California Institute of Technology, Pasadena, CA, USA

zhen.liu@jpl.nasa.gov

Eric J. Fielding

Jet Propulsion Laboratory, California Institute of Technology, Pasadena, CA, USA

eric.j.fielding@jpl.nasa.gov

Roland Bürgmann

Department of Earth and Planetary Science, Berkeley Seismological Laboratory, University of California at Berkeley, Berkeley, CA, USA

burgmann@seismo.berkeley.edu

This is a post-print submitted to EarthArXiv before final IEEE editing and formatting. The paper is published as:

C. Liang, Z. Liu, E. J. Fielding, and R. Bürgmann, "InSAR time series analysis of L-band wide-swath SAR data acquired by ALOS-2," IEEE Transactions on Geoscience and Remote Sensing, vol. 56, no. 8, pp. 4492-4506, Aug. 2018. DOI:

10.1109/TGRS.2018.2821150 
This is author's post-print before final IEEE editing and formatting. The paper is published as:

C. Liang, Z. Liu, E. J. Fielding, and R. Bürgmann, "InSAR time series analysis of L-band wide-swath SAR data acquired by ALOS-2," IEEE

Transactions on Geoscience and Remote Sensing, vol. 56, no. 8, pp. 4492-4506, Aug. 2018. DOI: 10.1109/TGRS.2018.2821150

\title{
InSAR Time Series Analysis of L-band Wide- Swath SAR Data Acquired by ALOS-2
}

\author{
Cunren Liang, Zhen Liu, Eric J. Fielding, and Roland Bürgmann
}

\begin{abstract}
Operating at L-band ( 24 cm wavelength) in wideswath modes is one of the characteristics of new and next generation satellite synthetic aperture radar (SAR) missions. After three years of operation, the JAXA ALOS-2 satellite has acquired a wealth of L-band wide-swath SAR data over many areas using its ScanSAR mode. We present SAR interferometry (InSAR) time series analysis results from ALOS-2 ScanSAR data. We analyze the possible error sources in the InSAR and correct them if possible. We present different time series analysis results including azimuth frequency modulation (FM) rate error, line of sight (LOS) ionospheric phase, azimuth shift caused by the ionosphere, and LOS displacement processed using both fullaperture and burst-by-burst workflows. The final InSAR LOS displacement time series result reveals both large-scale tectonic and small-scale anthropogenic deformation components. The results demonstrate the potential for measuring continental or even global-scale tectonic deformation and illustrate the promise of upcoming L-band wide-swath SAR missions, such as the NASA-ISRO SAR (NISAR) mission.
\end{abstract}

Index Terms-ALOS-2, InSAR time series analysis, L-band, ScanSAR, wide swath.

\section{INTRODUCTION}

$\mathrm{I}_{\mathrm{e}}^{\mathrm{N}}$ NSAR time series analysis aims to analyze the temporal evolution of the Earth's surface deformations with millimeter accuracy. Such deformation can be related to natural processes and hazards such as earthquakes, volcanoes, landslides, and glaciers, or caused by anthropogenic activities, such as groundwater and oil pumping, gas and geothermal extraction, mining, and urban subsidence. Permanent scatterer (PS) [1] and Small BAseline Subset (SBAS) [2] methods are the two most commonly used techniques for InSAR time series analysis. The InSAR data used in most previous time series analyses are characterized by short wavelength (C-band, $\sim 24 \mathrm{~cm}$ wavelength or X-band, $\sim 3 \mathrm{~cm}$ wavelength) or small

This research was carried out at the Jet Propulsion Laboratory (JPL), California Institute of Technology, under a contract with the National Aeronautics and Space Administration (NISAR Mission Science Definition Team Project, and NASA Earth Surface and Interior focus area). This research was also supported by an appointment to the NASA Postdoctoral Program at JPL.

Cunren Liang, Zhen Liu, and Eric J. Fielding are with the Jet Propulsion Laboratory, California Institute of Technology, Pasadena, CA 91109 USA.

Roland Bürgmann is with the Department of Earth and Planetary Science and Berkeley Seismological Laboratory, University of California, Berkeley, CA 94720 USA.

Copyright 2017 California Institute of Technology. U.S. Government sponsorship acknowledged. spatial coverage $(\sim 100 \mathrm{~km})$. These data are mostly acquired in the conventional stripmap mode by past and active satellite missions, such as the European Space Agency (ESA) ERS-1/2 and Envisat satellites, the Italian Space Agency COSMOSkyMed constellation, the German Aerospace Center (DLR) TerraSAR-X/TanDEM-X satellites, and the Japan Aerospace Exploration Agency (JAXA) ALOS-1 satellite. The short wavelength of most of these systems leads to strong decorrelation in the SAR interferogram, while the small coverage is not efficient for measuring large-scale tectonic deformation.

L-band ( $24 \mathrm{~cm}$ wavelength) can improve the InSAR coherence and make phase unwrapping easier. A wide-swath SAR can image a larger area and also help reduce the repeat time. Wide swath can be achieved by different techniques, such as ScanSAR [3], [4], TOPS [5], SweepSAR [6], and staggered SAR [7]. In ScanSAR and TOPS modes, a wide swath consists of several adjacent subswaths. The radar antenna is pointed to different subswaths to collect a burst at each subwath, which leads to reduced illumination time in azimuth direction and hence reduced azimuth resolution. In SweepSAR mode, the transmit beam illuminates a wide swath, but the receive beam is swept across the wide swath to track the radar echo. It is particularly suited for longer wavelength SARs. Another promising wide-swath mode is staggered SAR which images a wide swath by continuously changing pulse repetition interval in a cyclic manner. Compared with ScanSAR and TOPS, SweepSAR and staggered SAR can maintain high azimuth resolution. Wide swath is becoming one of the most important characteristics of the new generation satellite SAR missions and has taken the place of the conventional stripmap mode as the default acquisition mode for many new satellites. The Sentinel-1A/B, launched by ESA within the Copernicus program, achieves a swath width of $250 \mathrm{~km}$ by employing the TOPS mode. The JAXA ALOS-2 operates in ScanSAR mode with a $350 \mathrm{~km}$ swath width for global coverage, and is the first satellite SAR mission that routinely acquires L-band wide-swath data. The L-band wavelength and well-controlled orbit of ALOS-2 lead to significant improvement in InSAR coherence compared with previous missions. In the next few years, there will be a fleet of new satellite SAR missions joining the L-band wideswath SAR family. The NASA-ISRO SAR (NISAR) mission [8], currently planned to be launched in 2021, will use the novel SweepSAR technique [6] to achieve a swath width of $240 \mathrm{~km}$. The JAXA ALOS-4 mission is planned to continue to 
use ScanSAR mode to acquire data with $700 \mathrm{~km}$ swath width [9]. The DLR Tandem-L mission is expected to have a swath width of up to $350 \mathrm{~km}[10]$. With these new missions, we are going to enter a new era of L-band wide-swath mode.

InSAR analysis is one of the primary drivers of these current and future L-band wide-swath SAR missions. The great benefits brought to InSAR by combining L-band and wide swath have already been demonstrated by ALOS-2 (e.g. [11]). With L-band wide-swath data, it's easier to measure large-scale tectonic deformation, even in the areas that have low coherence in short-wavelength SAR data. In the meantime, small-scale deformation measurement is still possible depending on the resolution of the system. So far, not many comprehensive studies have considered InSAR time series analysis using wide-swath SAR data. In [12], InSAR time series analysis using ScanSAR data synthesized from Cband stripmap SAR data is evaluated. In [13], C-band ScanSAR data is used to improve the temporal resolution of the stripmap time series. In [14], the performance of TOPS time series is assessed with X-band TerraSAR-X data by comparing TOPS time series with stripmap time series. However, the wide swath, which is the important characteristic, is not evaluated in these studies. After the launch of Sentinel-1A/B, many time series analyses have been done using the C-band TOPS data (e.g. [15]), but are more or less application oriented.

ALOS-2 was launched on May 24, 2014 (UTC). After three years of operation, it has acquired a wealth of L-band wideswath data. InSAR processing methods, including the ionospheric correction technique, have been developed to process ALOS-2 ScanSAR data [11], [16], [17]. The ALOS-2 ScanSAR interferograms have made important contributions to the study of earthquakes, especially larger ones such as the $2015 \mathrm{Mw} 7.8$ Gorkha Earthquake in Nepal [11], [18] and the 2016 Mw 7.8 Kaikōura earthquake in New Zealand [19]. Time series analysis of the ALOS-2 ScanSAR data can benefit many more geoscience applications. In the following sections, we analyze the possible errors in ALOS-2 ScanSAR InSAR processing. We present a number of different time series results. The final mean deformation velocity derived from InSAR time series is validated by GPS measurements and reveals both large-scale tectonic deformation and small-scale anthropogenic deformation in an area spanning southern California and western Arizona, United States, and northern Baja California and Sonora, Mexico.

\section{DATA}

ALOS-2 can acquire data in spotlight, stripmap and ScanSAR modes. It has three wavelengths, different range bandwidths, different polarization modes, left/right looking ability, and different beams and coverage. Even in the same acquisition mode, these parameters might be different [20]. Among these different acquisition modes with different parameters, one ScanSAR mode is the default acquisition mode (hereafter referred to as default mode) for routine global coverage. Table I lists the important parameters of this default mode. This is the L-band wide-swath data that we use in our
InSAR time series analysis. For most of the areas, this should also be the data used for InSAR time series analysis. The Level 1.1 ScanSAR data that can be used for InSAR is distributed by JAXA in two different focused formats. In one format, the data is focused burst by burst, and individual burst images are directly available. In the other format, the data is focused by full-aperture algorithm [21], and the resulting image is a continuous stripmap-like image. Currently, the fullaperture product is usable for InSAR [22].

As a demonstration example, we process ALOS-2 ScanSAR data acquired on descending track 165 . The coverage of the data is shown in Fig. 1. Track 165 covers a large area centered on the "big bend" of the southern San Andreas-San Jacinto Fault system. Here, slip is distributed among multiple strikeslip faults, including the San Andreas (SAF), San Jacinto, Elsinore, and Superstition Hills Faults, which accommodate most of the North American-Pacific plate motion $(\sim 50 \mathrm{~mm} / \mathrm{yr})$ from south of Cajon Pass to Baja California [23], [24]. The track also spans the Eastern California Shear Zone to the east of the SAF, a $\sim 100-\mathrm{km}$-wide deformation zone in the Mojave Desert trending approximately N24 W. Several large historic earthquakes occurred in the Eastern California Shear Zone, including the recent $1992 \mathrm{Mw} 7.3$ Landers and $1999 \mathrm{Mw} 7.1$ Hector Mine earthquakes. The area is also rich in anthropogenic deformation signals due to non-tectonic sources, including groundwater extraction and replenishment, oil and gas extraction activity, and geothermal power generation. One such example is the Cerro Prieto Geothermal field in northern Baja California [25].

\section{INSAR PROCESSING, IONOSPHERIC CORRECTION AND ERROR ANALYSIS}

\section{A. InSAR processing and ionospheric correction}

We use two workflows, namely the full-aperture processing workflow and burst-by-burst processing workflow, to process the ALOS-2 InSAR data. For full-aperture processing [11], we use a multiband bandpass filter (MBF) to remove the nonoverlap spectrum caused by burst misalignment from the fullaperture images. We use incoherent cross-correlation to estimate the offsets between the master and slave images and fit a polynomial to the offsets in each direction (range and azimuth). The polynomials are used to resample the slave image to form the subswath interferograms. We also use incoherent cross-correlation to estimate the offsets between adjacent subswaths and use these offsets to mosaic the subswaths. After mosaicking, the processing is the same as stripmap InSAR processing.

For burst-by-burst processing [16], we extract the bursts from full-aperture images, during which the non-overlap spectrum caused by burst misalignment is also removed. We use a three-step method to do the coregistration. This includes geometrical coregistration, cross-correlation coregistration and spectral diversity coregistration. We also estimate the azimuth FM rate error using the spectral diversity interferograms and remove its effect [26]. For each pair of bursts, we resample the slave burst to form the burst interferogram. The burst 
interferograms are then mosaicked to form the subswath interferogram. The following steps are generally the same as full-aperture processing. We also use the methods in [16] to get the full-swath spectral diversity interferograms to measure the large-scale azimuth shifts caused by the ionosphere.

For each workflow, we use the range split-spectrum method [16], [27], [28] to do the ionospheric correction. For fullaperture processing, we filter the full-aperture images to get the subband images, while for burst-by-burst processing, we filter the burst images to get the subband images. For each subband, subswath interferograms are mosaicked to form the full-swath interferograms which are used to calculate the ionospheric phase. In addition to the subswath offsets calculated by cross correlation, we calculate phase differences between subswaths to further remove the effect of relative range time errors between subswaths.

Since currently the full-aperture product of ALOS-2 is usable for InSAR and it is easier to adapt a stripmap InSAR processor to full-aperture product, the full-aperture processing workflow is mostly used to process full-aperture product, but the standard way of ScanSAR InSAR processing is burst-byburst processing. The burst-by-burst processing also provides us more information such as azimuth measurement which contains ionospheric azimuth shift and azimuth deformation. Despite of the different processing procedures, the resulting interferograms of full-aperture processing and burst-by-burst processing should be equivalent [16], [29]. In Fig. 2, the two interferograms are indeed almost the same before ionospheric correction. After ionospheric correction, however, there are some differences. The differences are mainly caused by the differences of the ionospheric phases used for the ionospheric correction, which are calculated in the two processing workflows, respectively. The slight difference of the random noise level can cause the differences between the two ionospheric phases, since the total available range bandwidth for ionospheric correction is small. Other possible causes of the differences are analyzed in the following sections.

B. Effects of same and different phase errors in lower and upper band interferograms in ionospheric phase calculation

InSAR phase can be represented by [27], [28]

$$
\begin{aligned}
\Delta \phi & =\frac{4 \pi f_{0}}{c}\left(\Delta r_{\text {topo }}+\Delta r_{\text {motion }}+\Delta r_{\text {tropo }}\right)-\frac{4 \pi K}{c f_{0}} \Delta \text { TEC } \\
& =\Delta \phi_{\text {nd }}+\Delta \phi_{\text {ion }}
\end{aligned}
$$

where $c$ is the speed of light in vacuum, $f_{0}$ is the radar center frequency, $\Delta r_{\text {topo }}, \Delta r_{\text {motion }}$ and $\Delta r_{\text {tropo }}$ are differential twoway path delays caused by topography, ground motion and troposphere, respectively, $\triangle \mathrm{TEC}$ is the total electron content (TEC) difference along radar line of sight, $K=40.28 \mathrm{~m}^{3} / \mathrm{s}^{2}$, $\Delta \phi_{\text {nd }}$ is the non-dispersive component of the InSAR phase caused by topography, displacement and troposphere, and $\Delta \phi_{\text {ion }}$ is the dispersive component of the InSAR phase caused by the ionosphere. In the range split-spectrum method for ionospheric correction [27], [28], we create a lower band interferogram and an upper band interferogram with center frequencies $f_{l}$ and $f_{u}$, respectively. $\Delta r_{\text {topo }}, \Delta r_{\text {motion }}, \Delta r_{\text {tropo }}$, and $\triangle$ TEC remain unchanged regardless of radar frequency.
Thus, if we rewrite (1) with these frequencies, the phases of the two interferograms are

$$
\left\{\begin{array}{l}
\Delta \phi_{l}=\Delta \phi_{\text {nd }} \frac{f_{l}}{f_{0}}+\Delta \phi_{\text {ion }} \frac{f_{0}}{f_{l}} \\
\Delta \phi_{u}=\Delta \phi_{\text {nd }} \frac{f_{u}}{f_{0}}+\Delta \phi_{\text {ion }} \frac{f_{0}}{f_{u}}
\end{array}\right.
$$

If there are a common phase error $\Delta \phi_{\epsilon}$ in both interferograms, and an additional phase error $\Delta \phi_{\epsilon 2}$ in the upper band interferogram, the phases of the interferograms are

$$
\left\{\begin{array}{c}
\Delta \phi_{l}=\Delta \phi_{\text {nd }} \frac{f_{l}}{f_{0}}+\Delta \phi_{\text {ion }} \frac{f_{0}}{f_{l}}+\Delta \phi_{\epsilon} \\
\Delta \phi_{u}=\Delta \phi_{\text {nd }} \frac{f_{u}}{f_{0}}+\Delta \phi_{\text {ion }} \frac{f_{0}}{f_{u}}+\Delta \phi_{\epsilon}+\Delta \phi_{\epsilon 2}
\end{array}\right.
$$

Then the resulting calculated ionospheric phase is

$$
\begin{gathered}
\Delta \phi_{\text {ion }}=\frac{f_{l} f_{u}}{f_{0}\left(f_{u}^{2}-f_{l}^{2}\right)}\left(\Delta \phi_{l} f_{u}-\Delta \phi_{u} f_{l}\right) \\
-\frac{f_{l} f_{u}}{f_{0}\left(f_{u}^{2}-f_{l}^{2}\right)}\left[\Delta \phi_{\epsilon} f_{u}\right. \\
\left.-\left(\Delta \phi_{\epsilon}+\Delta \phi_{\epsilon 2}\right) f_{l}\right] \\
\approx \frac{f_{l} f_{u}}{f_{0}\left(f_{u}^{2}-f_{l}^{2}\right)}\left(\Delta \phi_{l} f_{u}-\Delta \phi_{u} f_{l}\right)-\frac{1}{2} \Delta \phi_{\epsilon} \\
+\frac{f_{u} f_{l}^{2}}{f_{0}\left(f_{u}^{2}-f_{l}^{2}\right)} \Delta \phi_{\epsilon 2}
\end{gathered}
$$

The first term in (4) is the correct ionospheric phase. The common phase error results in the second term in (4), while the additional phase error causes the third term in (4). Considering the narrow range bandwidth of the ALOS-2 ScanSAR mode (12 MHz actual bandwidth, Table I), we can see that the third term is significantly amplified by $\frac{f_{u} f_{l}^{2}}{f_{0}\left(f_{u}^{2}-f_{l}^{2}\right)}$. A special case of such error analysis is presented in [27] for analyzing the effects of phase unwrapping errors on ionospheric phase calculation.

\section{Effect of azimuth coregistration error on ionospheric phase calculation}

The Doppler frequency (including Doppler centroid frequency) can be represented by [30]

$$
f_{d}=\frac{2 v_{s} \sin \left(\theta_{\mathrm{sq}}\right)}{\lambda}=\frac{2 v_{s} \sin \left(\theta_{\mathrm{sq}}\right)}{c} f_{0}
$$

where $v_{s}$ is the satellite velocity on orbit, $\theta_{\text {sq }}$ is the squint angle, and $\lambda$ is the radar wavelength. In the azimuth direction, the linear phase in the SAR image is caused by the center Doppler frequency

$$
\phi(\eta)=2 \pi f_{d} \eta
$$

where $\eta$ is the azimuth time. In the cross-correlation coregistration, we fit a polynomial to the offsets, which usually contains errors due to azimuth shift caused by the ionosphere. If there is an azimuth coregistration error $\Delta \eta$ caused by ionospheric azimuth shift, the resulting phase error in InSAR phase is

$$
\Delta \phi_{\epsilon}^{\text {coreg }}=2 \pi f_{d} \Delta \eta
$$

We use the same offsets for resampling when generating the lower and upper band interferograms in the ionospheric phase calculation. For the two interferograms, all the variables in (5), 
except $f_{0}$, are the same. The range bandwidth of ALOS-2 ScanSAR mode is very small, that is, $f_{l} \approx f_{u}$. Therefore, the Doppler frequencies of the lower and upper bands are approximately the same, $f_{d, l} \approx f_{d, u}$. For the same coregistration error $\Delta \eta$, the resulting phase errors in the lower and upper band interferograms should be approximately the same, $\Delta \phi_{\epsilon, l}^{\text {coreg }} \approx \Delta \phi_{\epsilon, u}^{\text {coreg }}$. According to the result in Section III-B, since the resulting phase errors in the lower and upper band interferograms are the same, the error caused in the calculated ionospheric phase is approximately $-\frac{1}{2} \Delta \phi_{\epsilon, l}^{\text {coreg }}$.

In full-aperture processing, the effect of coregistration error on the InSAR phase also depends on the magnitude of the error. Fig. 3 shows interferograms with different coregistration errors. In Fig. 3(a) is the azimuth profile of a target focused using full-aperture algorithm. The result consists of a lot of spikes in the azimuth profile. The green envelope is the corresponding single burst focusing result. Fig. 3(b) shows the correct coregistration result. In Fig. 3(c), there is a coregistration error, but the same target in the other image is still coregistered on one side of the center spike. This is like the coregistration error in stripmap InSAR processing, and leads to some decorrelation and phase error in the interferogram, which is explained in [31]. In Fig. 3(d), the same target in the other image is coregistered to the foot or minimum of the center spike. The signal here is weak, and this leads to severe decorrelation. In Fig. 3(e), the same target in the other image is coregistered to another spike adjacent to the center spike. While the coherence in the resulting interferogram is still good, there is a large phase error in the interferogram.

\section{Effect of subswath phase inconsistency on ionospheric phase calculation}

Before calculating the ionospheric phase, we mosaic the subswath interferograms at each subband. The mosaicked subband interferograms are then used to calculate ionospheric phase. The coregistration error in each subswath leads to the subswath phase inconsistency in the mosaicked interferogram. In addition, the relative range time error of the subswaths is converted to phase error in the subband processing, which also causes subswath phase inconsistency [16]. The two phase errors in the lower and upper band interferograms are given by

$$
\left\{\begin{array}{l}
\Delta \phi_{\epsilon, l}=\Delta \phi_{\epsilon, l}^{\text {coreg }}+\Delta \phi_{\epsilon, l}^{\text {rg_time }} \\
\Delta \phi_{\epsilon, u}=\Delta \phi_{\epsilon, u}^{\text {coreg }}+\Delta \phi_{\epsilon, u}^{\text {rg_time }}
\end{array}\right.
$$

As discussed in Section III-C, for phase error caused by coregistration error, $\Delta \phi_{\epsilon, l}^{\text {coreg }}=\Delta \phi_{\epsilon, u}^{\text {coreg }}=\Delta \phi_{\epsilon}^{\text {coreg }} \quad$ (Same coregistration offsets used for generating regular, lower band and upper band interferograms). For phase error caused by relative range time error, $\Delta \phi_{\epsilon, l}^{\text {rg_time }}=-\Delta \phi_{\epsilon, u}^{\text {rgtime }}$. An example of subswath phase inconsistency caused by coregistration error in the regular interferogram in fullaperture processing is presented in Fig. 4. Fig. 4(a) is the spectral diversity interferogram of subswath 1 processed by burst-by-burst processing. It shows the complex pattern of azimuth shift caused by ionosphere, which may affect the cross-correlation coregistration in full-aperture processing. Fig. 4(b) is the interferogram processed by full-aperture processing, which shows the phase inconsistency between subswath 1 and subswath 2 . Azimuth offsets estimated by cross correlation are shown as functions of range sample number in Fig. 4(c) and azimuth line number in Fig.4(d). These offsets show the azimuth shift caused by the ionosphere, which causes coregistration error when fitting a low-order polynomial to the offsets.

To remove the effect of the relative range time error [16], we calculate the phase differences between adjacent subswaths, which can be given by

$$
\left\{\begin{array}{l}
\Delta \phi_{\mathrm{rm}, l}=\Delta \phi_{\epsilon}^{\text {coreg } \prime}+\Delta \phi_{\epsilon}^{\text {rg_time }}+\Delta \phi_{\epsilon, l}^{\text {noise }} \\
\Delta \phi_{\mathrm{rm}, u}=\Delta \phi_{\epsilon}^{\text {coreg }}-\Delta \phi_{\epsilon}^{\text {rg_time }}+\Delta \phi_{\epsilon, u}^{\text {noise }}
\end{array}\right.
$$

where $\Delta \phi_{\epsilon}^{\text {coreg' }}$ is part of the phase error caused by coregistration error, and $\Delta \phi_{\epsilon, l}^{\text {noise }}$ and $\Delta \phi_{\epsilon, u}^{\text {noise }}$ are the phase errors in the calculated phase difference caused by phase noise in the lower and upper band interferograms, respectively. Note that $\Delta \phi_{\epsilon}^{\text {coreg }}$ is varying across the whole subswath, but we calculate a constant phase difference in the small overlap between adjacent subswaths. Therefore, we use $\Delta \phi_{\epsilon}^{\text {coreg' }}$ instead of $\Delta \phi_{\epsilon}^{\text {coreg }}$ in (9).

After removing (9) from next subswath, the phase errors in the lower and upper band interferograms become

$$
\left\{\begin{array}{l}
\Delta \phi_{\epsilon, l}=\Delta \phi_{\epsilon, l}^{\text {coreg,adj }}+\Delta \phi_{\epsilon, l}^{\text {noise }} \\
\Delta \phi_{\epsilon, u}=\Delta \phi_{\epsilon, u}^{\text {coreg,adj }}+\Delta \phi_{\epsilon, u}^{\text {noise }}
\end{array}\right.
$$

where $\Delta \phi_{\epsilon, l}^{\text {coreg,adj }}=\Delta \phi_{\epsilon, u}^{\text {coreg,adj }}$, which does not cause significant phase error in the calculation of ionospheric phase according to Section III-B. However, $\Delta \phi_{\epsilon, l}^{\text {noise }}$ is not necessarily equal to $\Delta \phi_{\epsilon, u}^{\text {noise }}$. Furthermore, this error may accumulate as we apply phase adjustments from subswath 2 to subswath 5. Therefore, it may cause larger phase error in ionospheric phase calculation according to Section III-B.

\section{E. Effect of azimuth FM rate error on spectral diversity}

For ScanSAR, we use two bursts to form the spectral diversity interferogram. For a target, if we suppose that the time offsets between the centers of the burst signals and the zero-frequency time are $\eta_{c, l}$ and $\eta_{c, u}$, respectively, then $\eta_{c, u}-$ $\eta_{c, l}=n T_{C}$, where $n$ is the burst number difference and $T_{C}$ is the burst cycle length.

The spectral diversity interferogram is sensitive to the azimuth FM rate error of the matched filter in focusing. Supposing that the azimuth FM rate errors in the master and slave matched filters are $\Delta K_{m}$ and $\Delta K_{s}$, respectively, the phase error in the double-difference interferometric (DDI) phase used for spectral diversity (SD) is [26]

$$
\begin{aligned}
\phi_{\mathrm{SD}, \epsilon}(\eta) & =\pi\left(\Delta K_{s}-\Delta K_{m}\right)\left(\eta_{c, u}+\eta_{c, l}\right)\left(\eta_{c, u}-\eta_{c, l}\right) \\
& =2 \pi\left(\Delta K_{s}-\Delta K_{m}\right) n T_{C} \eta
\end{aligned}
$$

where $-\frac{1}{2}\left(T_{A}-n T_{C}\right)+\eta_{\mathrm{DC}} \leq \eta \leq \frac{1}{2}\left(T_{A}-n T_{C}\right)+\eta_{\mathrm{DC}}, T_{A}$ is the full-aperture length, and $\eta_{\mathrm{DC}}$ is the azimuth time corresponding to Doppler centroid frequency (origin: zerofrequency time). To obtain the final equality in (11), we have merged $\eta_{c, l}$ and $\eta_{c, u}$ into $\eta$, that is, $\eta_{c, u}+\eta_{c, l}=2 \eta$, as both of 
them are linearly proportional to the azimuth position of the target.

In spectral diversity coregistration, the error in the coregistration offset estimate is

$$
\Delta \eta_{\epsilon}=\frac{\phi_{\mathrm{SD}, \epsilon}(\eta)}{2 \pi \Delta f}=\frac{\phi_{\mathrm{SD}, \epsilon}(\eta)}{2 \pi n K_{a} T_{C}}=\frac{\Delta K_{s}-\Delta K_{m}}{K_{a}} \eta
$$

where $\Delta f$ is the difference of the two burst center frequencies and $K_{a}$ is the azimuth FM rate. We calculate this error as a function of the azimuth time and the azimuth FM rate error difference between the master and slave using parameters from an example scene, and the result is shown in Fig. 5.

\section{F. Tropospheric phase}

The tropospheric phase screen [32] due to water vapor variations can be very large across an area covered by a wideswath SAR image. An example is shown in Fig. 6, where there is more than $36 \mathrm{~cm}$ of apparent range change across the $350 \times 350 \mathrm{~km}$ ALOS-2 scene after applying the ionospheric correction described above. Acquisitions with very different tropospheric phase can be removed from the time series analysis, especially considering the low temporal resolution of ALOS-2 acquisitions in most locations. In high topographic relief areas such as the edges of the Tibetan Plateau [33], this may be a limitation for InSAR time series analysis with current data. In this case, more future acquisitions are required to measure the large-scale tectonic deformation. Alternatively, tropospheric phase can be mitigated by making use of weather models [34], GPS [35] and spectrometer observations [36], or the relationship between the interferometric phase and the topography [37]. In this study, we do not attempt to apply such tropospheric corrections.

\section{INSAR TIME SERIES ANALYSIS}

We use least squares throughout this section to estimate various things. Supposing that we have the following linear equation

$$
\mathbf{H} \boldsymbol{\theta}=\mathbf{s}
$$

where $\boldsymbol{\theta}$ is the vector of unknown parameters, $\mathbf{s}$ is the signal vector and $\mathbf{H}$ is the observation matrix. Due to observation noise or model inaccuracies, we observe a perturbed version of $\mathbf{s}$, which we denote by vector $\mathbf{x}$. The least squares estimation of $\boldsymbol{\theta}$ is found to be [38]

$$
\widehat{\boldsymbol{\theta}}_{\mathrm{LSE}}=\left(\mathbf{H}^{T} \mathbf{H}\right)^{-1} \mathbf{H}^{T} \mathbf{x}
$$

Supposing we have a weighting matrix $\mathbf{W}$, the weighted least squares estimation of $\boldsymbol{\theta}$ is [38]

$$
\widehat{\boldsymbol{\theta}}_{\mathrm{WLSE}}=\left(\mathbf{H}^{T} \mathbf{W} \mathbf{H}\right)^{-1} \mathbf{H}^{T} \mathbf{W} \mathbf{x}
$$

In InSAR, many measurements are relative measurements, that is, there is a constant offset $\Delta x$ in each of the observations. With this vector of offsets $\Delta \mathbf{x}$, the least squares estimation is

$$
\begin{aligned}
\widehat{\boldsymbol{\theta}}_{\mathrm{LSE}}^{\prime} & =\left(\mathbf{H}^{T} \mathbf{H}\right)^{-1} \mathbf{H}^{T}(\mathbf{x}+\Delta \mathbf{x}) \\
& =\left(\mathbf{H}^{T} \mathbf{H}\right)^{-1} \mathbf{H}^{T} \mathbf{x}+\left(\mathbf{H}^{T} \mathbf{H}\right)^{-1} \mathbf{H}^{T} \Delta \mathbf{x} \\
& =\widehat{\boldsymbol{\theta}}_{\mathrm{LSE}}+\left(\mathbf{H}^{T} \mathbf{H}\right)^{-1} \mathbf{H}^{T} \Delta \mathbf{x}
\end{aligned}
$$

This estimation is done in time domain for each pixel (spacetime estimation), resulting a series of estimated images at acquisition times. Here, $\left(\mathbf{H}^{T} \mathbf{H}\right)^{-1} \mathbf{H}^{T} \Delta \mathbf{x}$ is the same for each pixel, so each estimated image still has a constant offset.

The weighted least squares estimation is

$$
\begin{aligned}
\widehat{\boldsymbol{\theta}}_{\mathrm{WLSE}}^{\prime} & =\left(\mathbf{H}^{T} \mathbf{W H}\right)^{-1} \mathbf{H}^{T} \mathbf{W}(\mathbf{x}+\Delta \mathbf{x}) \\
& =\left(\mathbf{H}^{T} \mathbf{W H}\right)^{-1} \mathbf{H}^{T} \mathbf{W} \mathbf{x}+\left(\mathbf{H}^{T} \mathbf{W H}\right)^{-1} \mathbf{H}^{T} \mathbf{W} \Delta \mathbf{x} \\
& =\widehat{\boldsymbol{\theta}}_{\mathrm{WLSE}}+\left(\mathbf{H}^{T} \mathbf{W H}\right)^{-1} \mathbf{H}^{T} \mathbf{W} \Delta \mathbf{x}
\end{aligned}
$$

Each pixel may have a different weighting matrix $\mathbf{W}$, so the offset in each resulting image may be varying over the whole image. Therefore in time series analysis, when we are not able to determine $\Delta \mathbf{x}$, we simply use least squares estimation; otherwise, the weighted least squares estimation can be used.

\section{A. Spatial baseline}

The spatial separations of the ALOS-2 orbits are well controlled. According to our experience, the perpendicular baselines between orbits are usually smaller than $500 \mathrm{~m}$. This reduces the phase error introduced by DEM error in the differential interferogram, so we do not estimate DEM errors in this study. The perpendicular baselines of the ALOS-2 acquisitions used in this research are shown in Fig. 7, with the interferometric pairs used in the time series analysis shown as blue lines. Two scenes, acquired on 2016/11/24 and $2017 / 07 / 20$ were excluded from our final time series processing because we could see those scenes had large tropospheric phase variations.

\section{B. Burst overlap}

Due to an error in the calculation of latitude, the burst timing of ALOS-2 changed periodically after it was launched. As a result, the burst overlap depended on the acquisition date, and the chance of enough burst overlap was low before this problem was corrected. An online document provided by JAXA [39] describes how to search for InSAR pairs with high burst overlap. The error was fixed on February 8, 2015, after which the burst overlap of ALOS-2 ScanSAR mode has been well controlled. In [40], the cross correlation of two images is used to estimate the burst overlaps of data acquired after February 8, 2015. Statistical analysis shows that the standard deviation of the actual burst misalignment is $7 \%$, which is slightly better than the planned $10 \%$ burst misalignment. Furthermore, the burst misalignment shows a sinusoidal systematic error with a cycle of approximately one year after the February 2015 correction.

For the ALOS-2 frame and track used in this research, while there are several scenes acquired before February 8, 2015, none of them have enough burst overlap with scenes acquired after this date according to the methods provided by JAXA [39]. Therefore, we ordered and used only 17 scenes (15 used in final time series analysis after excluding two scenes with large tropospheric phase variations) acquired after February 8, 2015. We use a standard method to calculate burst overlap [11]. First, we estimate the start times of raw bursts. We use a DEM and orbit data to calculate the azimuth offset between the InSAR pair and align the InSAR pair in the azimuth direction using the azimuth offset. The burst overlap can then be calculated using the start times of raw bursts. Fig. 8 shows the histogram of the calculated burst overlaps for the pairs used in the time series analysis. The burst overlap is good 
enough for interferometry in every pair, and excellent in most of the pairs.

\section{Azimuth FM rate error}

The DDI phase of the spectral diversity interferogram can be used to estimate the azimuth FM rate error. To suppress the strong noise in the DDI phase, we compute the average DDI phase within an area and get a column of average DDI phase, which is a function of azimuth time. We then fit a linear polynomial to the average DDI phase, and the azimuth FM rate error can be calculated as

$$
\Delta K_{s}-\Delta K_{m}=\frac{a}{2 \pi n T_{C}}
$$

where $a$ is the slope of the polynomial. The standard deviation of the estimated azimuth FM rate error is

$$
\sigma_{\Delta K_{S}-\Delta K_{m}}=\frac{1}{2 \pi n T_{C}} \sigma_{a}
$$

where $\sigma_{a}$ is the standard deviation of $a$. The problem of using this method to estimate azimuth FM rate error is that it can be biased when there is strong azimuth deformation or azimuth shift caused by ionosphere.

The whole imaged area is divided into a number of smaller areas. In each small area of each InSAR pair, we can get three azimuth FM rate error estimates with burst number difference $n$ ranging from 1 to 3 . We assume the azimuth FM rate error of the first acquisition (150219) is zero, and use the weighted least squares estimation in (15) to estimate the azimuth FM rate errors of the remaining acquisitions in each small area. We use (19) to compute the weighting matrix. For each acquisition, we can get a number of azimuth FM rate error estimates. We then fit a polynomial to the azimuth FM rate error estimates for all the patches and compute an azimuth FM rate error image using the polynomial for this acquisition. The results are shown in Fig. 9. Overall, the azimuth FM rate errors of these products are not very big. Note that the result of 161013 is biased due to the strong azimuth shift caused by the ionosphere which affects the estimation process.

\section{Line of sight (LOS) ionospheric phase}

We calculate the ionospheric phase for each InSAR pair using the range split-spectrum method [16]. Note that the calculated ionospheric phase is just the relative differential ionospheric phase of master and slave acquisitions, since both lower and upper band interferograms used to calculate ionospheric phase only have relative phases. Therefore, each ionospheric phase image has a constant offset. For the time series analysis of LOS deformation, we can find a stable area where there is no deformation and get the absolute deformations before doing time series analysis. For ionosphere, however, we cannot find an area where ionosphere is always stable. Therefore, we cannot use the weighted least squares estimation to estimate the ionospheric phase.

Instead, we use the least squares estimation in (14) to estimate the ionospheric phase for each acquisition. The original ionospheric phase maps are from full-aperture processing. We assume the ionospheric phase of the first acquisition is zero. The estimated ionospheric phases are shown in Fig. 10. We can see the strong ionospheric variations, reaching several meters across the wide-swath scene. According to our experience, this is common for ALOS-2 acquisitions. Ionospheric phase must be removed before InSAR time series analysis, especially for wide-swath mode.

\section{E. Azimuth shift caused by ionosphere}

We use the burst-by-burst processing to get the full-swath spectral diversity interferogram of each InSAR pair. We remove the phase errors caused by azimuth FM rate errors in the spectral diversity interferograms. For each InSAR pair, we can get three spectral diversity interferograms with burst number difference $n$ ranging from 1 to 3 . The three spectral diversity interferograms are unwrapped and converted to azimuth deformation images. Similar to the InSAR LOS deformation images, each azimuth deformation image has a constant offset. The final azimuth deformation image is the weighted average of the three azimuth deformation images (the weight of each image is constant) and therefore also has a constant offset. This is the relative differential azimuth shift of master and slave acquisitions caused by the ionosphere.

Since we cannot find an area where ionospheric shift is always the same to remove the offsets, we use the least squares estimation in (14) to estimate the azimuth shift for each acquisition. The results are shown in Fig. 11. These results show that the azimuth shift caused by the ionosphere usually involves a slowly changing shift with occasional streak-like features. Within the $350 \mathrm{~km}$ swath coverage, the amplitude of the large-scale azimuth shift is on the order of 4 $\mathrm{m}$.

\section{F. LOS deformation}

The workflows described in section III-A are used to process the SAR data. We select an area in the northeast corner of the imaged area as the reference area, which is known to be stable and is located east of the Eastern California Shear Zone. We use the weighted least squares estimation in (15) to estimate the range displacement of each acquisition. Since the InSAR phase is spatially filtered, we don't compute the phase standard deviation using coherence, but simply use coherence as weight. We use a Gaussian filter in the time domain to suppress tropospheric signals. We then estimate the mean LOS velocity using the estimated range displacements at each pixel. We estimate the mean velocity using full-aperture interferograms and burst-by-burst interferograms, both with ionospheric corrections applied. In addition, we also estimate the mean velocity using full-aperture interferograms without ionospheric corrections. Note that these are all what we do for time series analysis. We don't use more sophisticated techniques to improve the time series results because the resulting improvements often depend on the specific techniques, software, user experience and so on. Our focus here is to examine what accuracy we can achieve with the current ALOS-2 ScanSAR archive data. Importantly, no ramp removal or GPS calibration is done to any of the 
interferograms or the resulting mean velocity images.

The mean velocity maps derived from full-aperture interferograms without ionospheric corrections, full-aperture interferograms with ionospheric corrections, and burst-byburst interferograms with ionospheric corrections are shown in Figs. 12-14, respectively. We can see the strong ramp caused by the ionosphere in Fig. 12. The difference between mean velocity maps derived from full-aperture interferograms with ionospheric corrections and burst-by-burst interferograms with ionospheric corrections is shown in Fig. 15. The difference between the two workflow results can be explained in Section III. The main difference is caused by different ionospheric corrections in the two workflows. In particular, the main difference gradually changes from subswath 1 to subswath 5 , as the phase errors $\Delta \phi_{\epsilon, l}^{\text {noise }}$ and $\Delta \phi_{\epsilon, u}^{\text {noise }}$ in (10) accumulate from subswath 1 to subswath 5 . There is also a sharp but small discontinuity between subswath 1 and subswath 2 in Fig. 15 . This is related to phase errors in the full-aperture interferograms. The phase errors are the result of coregistration errors as shown in Fig. 4(b), as the azimuth shift caused by the ionosphere have affected the cross-correlation coregistration.

Over the large area covered by this ScanSAR frame, there are 466 GPS velocity estimates from the Southern California Earthquake Center (SCEC) Crustal Motion Map 4 (CMM4) [41] that can be compared with the InSAR velocity map. We project the 3-D GPS velocity measurements from CMM4 into the SAR LOS directions and compare them with the InSAR velocities estimated from the time series analysis. In Fig. 16, the vertical GPS velocities are set to zero before the projection (hereafter referred to as 2-D), while in Fig. 17, all the 3-D GPS velocity measurements are used (hereafter referred to as 3-D). In both figures, the blue crosses are evenly distributed near the one-to-one reference line indicating good agreement between the two LOS velocity estimates. In most InSAR studies of interseismic deformation, a long-wavelength ramp in the InSAR velocity map causes a systematic deviation from GPS velocities and has to be removed (e.g. [42]). Here, no systematic deviation from the diagonal line is observed in either figure indicating very small long-wavelength errors in our InSAR velocity field. The 2-D root mean square (RMS) error is lower than the 3-D RMS error, likely caused partly by the less accurate GPS vertical measurements. The GPS observation periods do not match the InSAR observation period, and there are likely still residual postseismic deformations in the data [43]. These may contribute to the RMS. Therefore, we also compared the InSAR velocities with continuous GPS data processed by the Nevada Geodetic Laboratory (http://geodesy.unr.edu/). 237 GPS velocities in the InSAR observation period are used for comparison. The 2D RMS and 3-D RMS are $4.25 \mathrm{~mm} / \mathrm{yr}$ and $4.35 \mathrm{~mm} / \mathrm{yr}$, respectively. This is an indication that the less accurate GPS vertical measurements lead to the larger 3-D RMS error. We also did the same comparisons with the GPS velocities from CMM4 using InSAR velocity map processed using burst-byburst interferograms, and the results are shown in Fig. 18 and Fig. 19, respectively. The resulting RMS errors are slightly higher but overall the two InSAR velocity maps are very similar.

The InSAR LOS velocity map processed using full-aperture interferograms along with GPS horizontal velocity vectors from CMM4 are shown in Fig. 20. We compare InSAR and GPS velocities projected into the SAR LOS along two profiles $A A^{\prime}$ and $B B^{\prime}$ (locations in Fig. 20) in Fig. 21 and Fig. 22, respectively. We can see that the InSAR and GPS measurements are in good agreement. The profile $A A^{\prime}$ spans the interseismic deformation across the eastern California shear zone. The profile $B B^{\prime}$ records the dominant interseismic deformation across the southern San Andreas fault-San Jacinto fault system and the southern Mojave Desert. There is no apparent residual ramp in the InSAR LOS deformation map as compared to the projected GPS velocities in both profiles. Based on the comparison between InSAR and GPS velocities in Figs. 16-22, we conclude that the large-scale tectonic deformation across the entire ALOS-2 ScanSAR frame can be measured with good accuracy through InSAR time series analysis.

We also observe the large local deformation signal at the Cerro Prieto Geothermal Field in Baja California in the southeast corner of Fig. 20, which is related to the sustained anthropogenic fluid extraction. In this area surrounded by agriculture of the Mexicali Valley, the coherence is lower, which affects the accuracies of both InSAR and ionospheric correction. The maximum vertical deformation from time series analysis is about $110 \mathrm{~mm} / \mathrm{yr}$, consistent with previous field and InSAR measurements [44].

Since ALOS-2 does not always acquire SAR data on each orbit over a site, measuring the short-term temporal evolution of the deformation signal is challenging, but we do explore time. Over this area, there were 17 ScanSAR scenes acquired between February 2015, when the ScanSAR burst synchronization was stabilized, and August 2017, roughly one scene every two months. We anticipate for the regions that have more frequent data acquisitions such as Japan, a reduction of tropospheric signal in the mean LOS velocity field can be achieved and the investigation of time-dependent deformation processes is possible.

\section{IMPLICATIONS FOR NISAR}

The NASA-ISRO SAR (NISAR) mission [8], expected to be launched in 2021, will fly a dual L- and S-band SAR. It uses the SweepSAR technique [6] to achieve wide-swath coverage and short repeat time. The L-band radar is planned to acquire data globally. It is designed to have an extra range band for ionospheric correction using the range split-spectrum method [28]. While there are a number of different acquisition modes (e.g. different range bandwidths and polarizations) for different application purposes in different areas, NISAR has a basic acquisition mode for global coverage (hereafter referred to as default mode). Comparison of this default mode and the ALOS-2 default mode is shown in Table II. Our following discussions focus on these two default acquisition modes of the L-band radars. 


\section{A. Comparison between ALOS-2 and NISAR}

Compared with ALOS-2, NISAR has an extra range band. This, together with the larger range bandwidth and better azimuth resolution, will improve the accuracy of ionospheric correction using the range split-spectrum method [45]. According to our experience, ALOS-2 data generally have good coherence globally, but are still subject to relatively strong decorrelation in areas such as agricultural areas. Both the range and azimuth resolutions of NISAR are better than ALOS-2, so we expect to lower the phase noise level with NISAR data in these areas. The repeat time of ALOS-2 is 14 days, but ALOS-2 does not always acquire data, which leads to an actual repeat imaging time of about two months or longer in most places. In some areas, currently there are only few acquisitions, which limits its use in time series analysis. In addition, ALOS-2 mainly acquires data on descending tracks. In comparison, the repeat time of NISAR is 12 days with more frequent acquisitions (every cycle) planned for both ascending and descending tracks. The shorter repeat cycle enables better reduction of the tropospheric contributions in the time series analysis and tracking the short-term temporal evolution of the deformations. For measuring large-scale tectonic motions, NISAR has a smaller swath width $(240 \mathrm{~km})$ than ALOS-2 $(350 \mathrm{~km})$, so more tracks will be required to cover large areas. The nominal mission duration of NISAR is 3 years, shorter than the planned 5-year duration of ALOS-2. This could limit its use to measure longer-term tectonic deformations, but NISAR will carry a fuel reserve that we hope can extend the mission.

\section{B. Combination of ALOS-2 and NISAR}

The ALOS-2 was launched on May 24, 2014 (UTC), and the goal of the mission duration is 7 years [46]. NISAR is expected to be launched near the end of the ALOS-2 mission. The look angle ranges of the two missions are close, but the satellite tracks might be different. Methods can be explored to combine the highly coherent L-band wide-swath SAR data acquired by the two missions. For example, the fault moving directions can be used as input when jointly making use of these data. Thus, we can have at least 10 years of data to look at the long-term global tectonic deformation.

\section{Coregistration of NISAR images}

From the time series analysis of the spectral diversity interferograms, we have shown that the azimuth shift caused by ionosphere is on the order of $4 \mathrm{~m}$. Note that the azimuth resolution of NISAR is about $7 \mathrm{~m}$. Therefore, for the accurate coregistration of NISAR images, ionospheric shift should be considered. Here, we propose the following coregistration strategy. First, we apply a geometrical coregistration [47]. We then do spectral diversity or cross-correlation coregistration to remove the possible constant offset and azimuth shift caused by the ionosphere. Spectral diversity is faster, but needs phase unwrapping and only measures relative offset. Cross correlation is slower, but is able to measure absolute offset. To improve the efficiency, we can leverage high performance computing tools such as GPU to accelerate cross correlation.
Another choice is the combination of the two. For example, we can measure the relative azimuth shift using spectral diversity for the whole image, and determine the absolute azimuth shift using cross correlation in a small area.

\section{CONCLUSION}

After three years of operation, ALOS-2 has acquired excellent L-band wide-swath SAR data for InSAR time series analysis in many areas using its ScanSAR mode. These acquisitions have small baselines and good burst alignment, which, along with L-band wavelength, lead to much better coherence. Over many interesting areas, ALOS-2 acquires a ScanSAR scene about every two months, but in many other areas there are only few acquisitions. Generally, mean velocity can be measured with these data. However, measuring the short-term temporal evolution of the deformation is still challenging over most areas. In areas with few acquisitions, even mean velocity measurement is difficult.

We find that it is crucial to correct ionospheric phase for accurately measuring LOS deformation. In addition, within the $350 \mathrm{~km}$ swath width of ALOS-2 ScanSAR mode, the azimuth shift caused by the ionosphere is on the order of four meters. This may cause problems in coregistration, especially for SAR data with high azimuth resolution. Our time series analysis of interferograms spanning the SAF plate boundary zone in southern California, processed by both full-aperture and burstby-burst workflows, reveals both large-scale tectonic and small-scale anthropogenic deformations. In particular, we show that there is no need for ramp error correction for the interferograms or the mean velocity. Our case study demonstrates the potential of using L-band wide-swath SAR data acquired by ALOS-2 for measuring continental or even global tectonic deformation.

Our pilot study of InSAR time series analysis with ALOS-2 can provide guidance for scientific exploitation of future Lband missions such as NISAR, ALOS-4, and Tandem-L. Furthermore, combining the L-band wide-swath SAR data acquired by ALOS-2 and future missions may allow us to study the long term deformation signals over continental or global scales.

\section{ACKNOWLEDGMENT}

The ALOS-2 original data are copyright JAXA and provided under JAXA RA4 PI project 1329. Fig. 20 is plotted using the GMT software [48].

\section{REFERENCES}

[1] A. Ferretti, C. Prati, and F. Rocca, "Permanent scatterers in SAR interferometry," IEEE Trans. Geosci. Remote Sens., vol. 39, no. 1, pp. 820, Jan. 2001.

[2] P. Berardino, G. Fornaro, R. Lanari, and E. Sansosti, "A new algorithm for surface deformation monitoring based on small baseline differential SAR interferograms," IEEE Trans. Geosci. Remote Sens., vol. 40, no. 11, pp. 2375-2383, Nov. 2002.

[3] K. Tomiyasu, "Conceptual performance of a satellite borne, wide swath synthetic aperture radar," IEEE Trans. Geosci. Remote Sens., vol. GE19, no. 2, pp. 108-116, Apr. 1981.

[4] R. K. Moore, J. P. Claassen, and Y. H. Lin, "Scanning spaceborne synthetic aperture radar with integrated radiometer," IEEE Trans. Aerosp. Electron. Syst., vol. AES-17, no. 3, pp. 410-420, May 1981. 
[5] F. De Zan and A. Monti Guarnieri, "TOPSAR: Terrain observation by progressive scans," IEEE Trans. Geosci. Remote Sens., vol. 44, no. 9, pp. 2352-2360, Sep. 2006.

[6] A. Freeman, G. Krieger, P. Rosen, M. Younis, W. T. K. Johnson, S. Huber, R. Jordan, and A. Moreira, "SweepSAR: Beam-forming on receive using a reflector-phased array feed combination for spaceborne SAR," in Proc. 2009 IEEE Radar Conference, Pasadena, CA, USA, May 4-8, 2009.

[7] M. Villano, G. Krieger, and A. Moreira, "Staggered SAR: highresolution wide-swath imaging by continuous PRI variation," IEEE Trans. Geosci. Remote Sens., vol. 52, no. 7, pp. 4462-4479, Jul. 2014.

[8] P. A. Rosen, S. Hensley, S. Shaffer, L. Veilleux, M. Chakraborty, T. Misra, R. Bhan, V. Raju Sagi, and R. Satish, "The NASA-ISRO SAR mission - An international space partnership for science and societal benefit," in Proc. 2015 IEEE Radar Conference, Arlington, VA, USA, May 10-15, 2015, pp. 1610-1613.

[9] http://global.jaxa.jp/projects/sat/alos4/ (last access date: Nov. 20, 2017)

[10] M. Eineder, I. Hajnsek, G. Krieger, A. Moreira, and K. Papathanassiou, "Tandem-L: Satellite mission proposal for monitoring dynamic processes on the Earth's surface," Tandem-L brochure, Apr. 2016. (can be downloaded from: http://www.dlr.de/hr/en/Portaldata/32/Resources/dokumente/tdml/Tande m-L Brochure 2017-05.pdf, last access date: Nov. 20, 2017)

[11] C. Liang and E. J. Fielding, "Interferometry with ALOS-2 full-aperture ScanSAR data," IEEE Trans. Geosci. Remote Sens., vol. 55, no. 5, pp. 2739-2750, May 2017.

[12] S. M. Buckley and K. Gudipati, "Evaluating ScanSAR interferometry deformation time series using bursted stripmap data," IEEE Trans. Geosci. Remote Sens., vol. 49, no. 6, pp. 2335-2342, Jun. 2011.

[13] A. Pepe, A. B. Ortiz, P. R. Lundgren, P. A. Rosen, and Riccardo Lanari, "The stripmap-ScanSAR SBAS approach to fill gaps in stripmap deformation time series with ScanSAR data," IEEE Trans. Geosci. Remote Sens., vol. 49, no. 12, pp. 4788-4804, Dec. 2011.

[14] M. Nannini, P. Prats-Iraola, F. De Zan, and D. Geudtner, "TOPS time series performance assessment with TerraSAR-X data," IEEE J. Sel. Topics Appl. Earth Observ. Remote Sens., vol. 9, no. 8, pp. 3832-3848, Aug. 2016.

[15] M. Shirzaei, R. Bürgmann, and E. J. Fielding, “Applicability of Sentinel1 Terrain Observation by Progressive Scans multitemporal interferometry for monitoring slow ground motions in the San Francisco Bay Area," Geophys. Res. Lett., vol. 44, no. 6, pp. 2733-2742, Mar. 2017.

[16] C. Liang and E. J. Fielding, "Measuring azimuth deformation with Lband ALOS-2 ScanSAR interferometry," IEEE Trans. Geosci. Remote Sens., vol. 55, no. 5, pp. 2725-2738, May 2017.

[17] R. Natsuaki, H. Nagai, T. Motohka, M. Ohki, M. Watanabe, R. B. Thapa, T. Tadono, M. Shimada, and S. Suzuki, "SAR interferometry using ALOS-2 PALSAR-2 data for the Mw 7.8 Gorkha, Nepal earthquake," Earth, Planets and Space, vol. 68, pp. 15, Feb. 2016.

[18] H. Yue et al., "Depth varying rupture properties during the $2015 \mathrm{Mw} 7.8$ Gorkha (Nepal) earthquake," Tectonophysics, vol. 714-715, pp. 44-54, Sep. 2017.

[19] I. J. Hamling et al., "Complex multifault rupture during the $2016 \mathrm{Mw}$ 7.8 Kaikōura earthquake, New Zealand," Science, vol. 356, no. 6334, Apr. 2017.

[20] Y. Kankaku, "ALOS-2 and PALSAR-2 development status," presented at the Cal-Val Sci. Team (CVST) Meeting.

[21] R. Bamler and M. Eineder, "ScanSAR processing using standard high precision SAR algorithms," IEEE Trans. Geosci. Remote Sens., vol. 34, no. 1, pp. 212-218, Jan. 1996.

[22] Personal communication, JAXA.

[23] C. DeMets and T. H. Dixon, "New kinematic models for Pacific-North America motion from $3 \mathrm{Ma}$ to present, I: Evidence for steady motion and biases in the NUVEL-1A Model," Geophys. Res. Lett., vol. 26, no. 13, pp. 1921-1924, Jul. 1999

[24] P. Lundgren, E. A. Hetland, Z. Liu and E. J. Fielding, "Southern San Andreas-San Jacinto fault system slip rates estimated from earthquake cycle models constrained by GPS and interferometric synthetic aperture radar observations," J. Geophys. Res., vol. 114, no. B2, Feb. 2009.

[25] X. Xu, D. T. Sandwell, E. Tymofyeyeva, A. González-Ortega, and X. Tong, "Tectonic and anthropogenic deformation at the Cerro Prieto geothermal step-over revealed by Sentinel-1A InSAR," IEEE Trans. Geosci. Remote Sens., vol. 55, no. 9, pp. 5284-5292, Jun. 2017.

[26] C. Liang, E. J. Fielding, and M.-H. Huang, "Estimating azimuth offset with double-difference interferometric phase: The effect of azimuth FM rate error in focusing," IEEE Trans. Geosci. Remote Sens., vol. 55, no. 12, pp. 7018-7031, Dec. 2017.

[27] G. Gomba, A. Parizzi, F. de Zan, M. Eineder, and R. Bamler, "Toward operational compensation of ionospheric effects in SAR interferograms: The split-spectrum method," IEEE Trans. Geosci. Remote Sens., vol. 54, no. 3, pp. 1446-1461, Mar. 2016.

[28] P. A. Rosen, S. Hensley, and C. Chen, "Measurement and mitigation of the ionosphere in L-band interferometric SAR data," in Proc. IEEE Radar Conf., Washington, DC, USA, May 2010, pp. 1459-1463.

[29] C. Liang, Q. Zeng, and J. Jiao, "An assessment of ScanSAR interferometric processing using full-aperture approach," IEEE Geosci. Remote Sens. Lett., vol. 11, no. 9, pp. 1559-1563, Sep. 2014.

[30] I. G. Cumming and F. H. Wong, Digital Processing of Synthetic Aperture Radar Data: Algorithms and Implementation. Norwood, MA, USA: Artech House, 2005.

[31] C. Liang and E. J. Fielding, "Interferometric processing of ScanSAR data using stripmap processor: New insights from coregistration," IEEE Trans. Geosci. Remote Sens., vol. 54, no. 7, pp. 4343-4354, Jul. 2016.

[32] D. P. S. Bekaert, R. J. Walters, T. J. Wright, A. J. Hooper, and D. J. Parker, "Statistical comparison of InSAR tropospheric correction techniques," Remote Sens. Environ., vol. 170, pp. 40-47, Dec. 2015.

[33] E. Fielding, B. Isacks, M. Barazangi, and C. Duncan, "How flat is Tibet?" Geology, vol. 22, no. 2, pp. 163-167, Feb. 1994.

[34] R. Jolivet, P. S. Agram, N. Y. Lin, M. Simons, M.-P. Doin, G. Peltzer, and $\mathrm{Z}$. Li, "Improving InSAR geodesy using Global Atmospheric Models," J. Geophys. Res., vol. 119, no. 3, pp. 2324-2341, Mar. 2014.

[35] C. Yu, N. T. Penna, and Z. Li, "Generation of real-time mode highresolution water vapor fields from GPS observations," J. Geophys. Res., vol. 122, no. 3, pp. 2008-2025, Feb. 2017.

[36] Z. Li, E. J. Fielding, P. Cross, and J.-P. Muller, "Interferometric synthetic aperture radar atmospheric correction: Medium Resolution Imaging Spectrometer and Advanced Synthetic Aperture Radar integration," Geophys. Res. Lett., vol. 33, no. 6, Mar. 2006.

[37] Y. N. Lin, M. Simons, E. A. Hetland, P. Muse, and C. DiCaprio, “A multiscale approach to estimating topographically correlated propagation delays in radar interferograms," Geochem. Geophys. Geosyst., vol. 11, no. 9, Sep. 2010.

[38] S. M. Kay, Fundamentals of Statistical Signal Processing: Estimation Theory. Upper Saddle River, NJ, USA: Prentice Hall, 1993.

[39] JAXA, Effective data for interferometric analysis with PALSAR-2 ScanSAR mode. (after login, can be downloaded from: https://auig2.jaxa.jp/openam/UI/Login?goto=http $\% 3 \mathrm{~A} \% 2 \mathrm{~F} \% 2 \mathrm{Fal} 2 \mathrm{mwb}$ 01\%3A80\%2Fips\%2Fhome, last access date: Nov. 20, 2017)

[40] R. Natsuaki, T. Motohka, M. Shimada, and S. Suzuki, "Burst misalignment evaluation for ALOS-2 PALSAR-2 ScanSAR-ScanSAR interferometry," Remote Sens., vol. 9, no. 3, Mar. 2017.

[41] Z.-K. Shen, R. W. King, D. C. Agnew, M. Wang, T. A. Herring, D. Dong, and P. Fang, "A unified analysis of crustal motion in Southern California, 1970-2004: The SCEC crustal motion map," J. Geophys. Res., vol. 116, no. B11, Nov. 2011.

[42] X. Tong, D. T. Sandwell, and B. Smith-Konter, "High-resolution interseismic velocity data along the San Andreas Fault from GPS and InSAR," J. Geophys. Res., vol. 118, no. 1, pp. 369-389, Jan. 2013.

[43] S. Liu, Z.-K. Shen, and R. Bürgmann, "Recovery of secular deformation field of Mojave Shear Zone in Southern California from historical terrestrial and GPS measurements," J. Geophys. Res., vol. 120, no. 5, pp. 3965-3990, May 2015.

[44] D. T. Trugman, A. A. Borsa, and D. T. Sandwell, "Did stresses from the Cerro Prieto Geothermal Field influence the El Mayor-Cucapah rupture sequence?" Geophys. Res. Lett., vol. 41, no. 24, pp. 8767-8774, Dec. 2014.

[45] H. Fattahi, M. Simons, and P. Agram, "InSAR time-series estimation of the ionospheric phase delay: An extension of the split range-spectrum technique," IEEE Trans. Geosci. Remote Sens., DOI: 10.1109/TGRS.2017.2718566.

[46] Y. Kankaku, Y. Osawa, S. Suzuki, and T. Watanabe, "The overview of the L-band SAR onboard ALOS-2," in Proc. PIERS, Moscow, Russia, Aug. 2009, pp. 735-738.

[47] E. Sansosti, P. Berardino, M. Manunta, F. Serafino, and G. Fornaro, "Geometrical SAR image registration," IEEE Trans. Geosci. Remote Sens., vol. 44, no. 10, pp. 2861-2870, Oct. 2006.

[48] P. Wessel, W. H. F. Smith, R. Scharroo, J. Luis, and F. Wobbe, "Generic mapping tools: Improved version released," Eos Trans. AGU, vol. 94, no. 45 , pp. 409-410, Nov. 2013. 


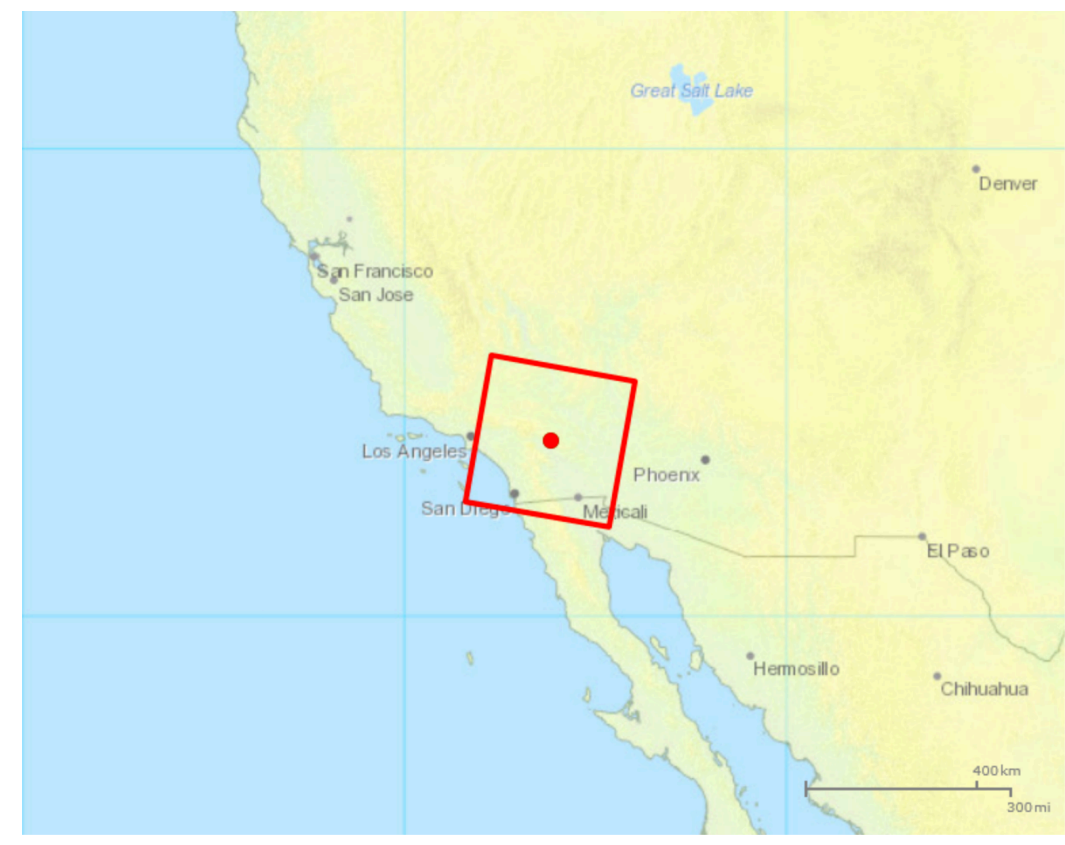

Fig. 1. Coverage of the ALOS-2 ScanSAR data used for InSAR time series analysis.
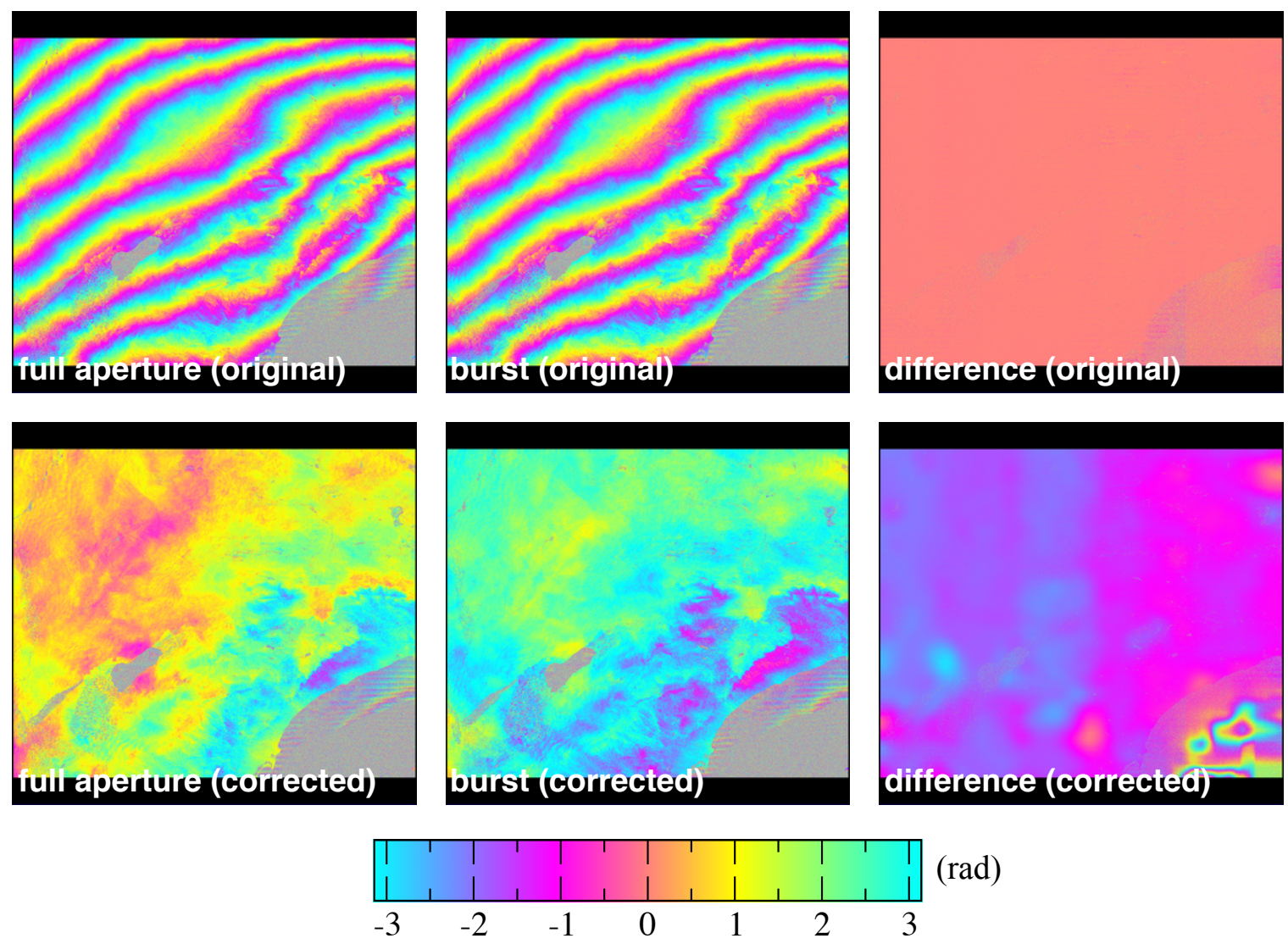

Fig. 2. The first row shows the original interferograms processed by full-aperture and burst-by-burst approaches and their difference. The second row shows the interferograms with ionospheric correction and their difference. The lower right area is ocean. The 
fringes on the ocean are caused by the sidelobes of the radar antenna. The discontinuities of the fringes on the ocean are mainly caused by filtering which is used to extract individual bursts or remove the non-overlap spectrum caused by burst misalignment, as the filtering parameters are defined for the mainlobe. Data: 150219-150402.

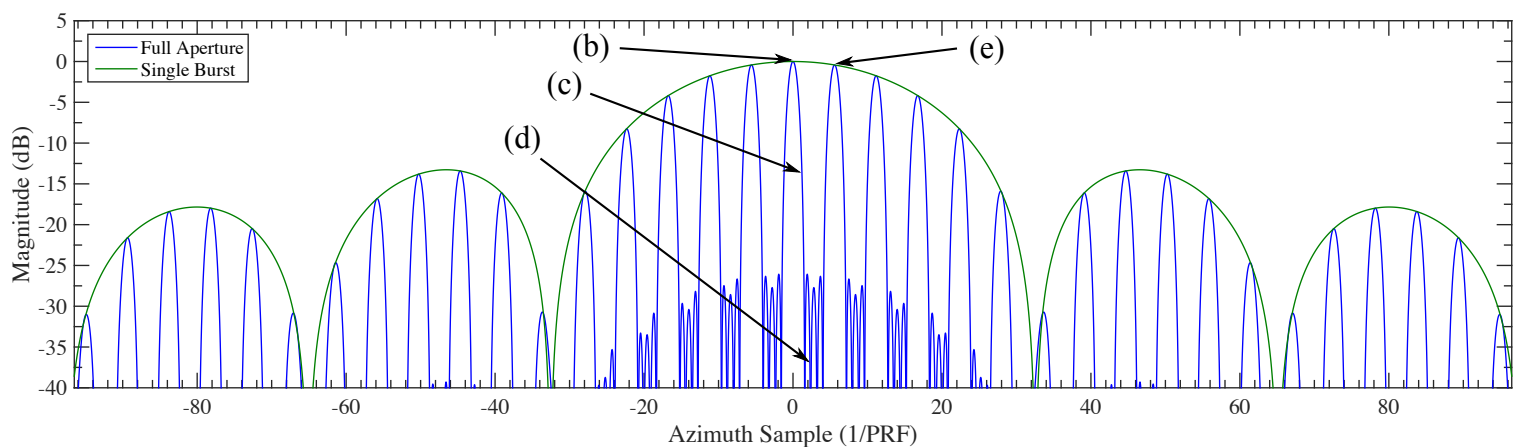

(a)

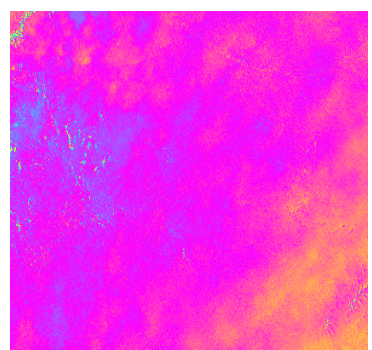

(b)

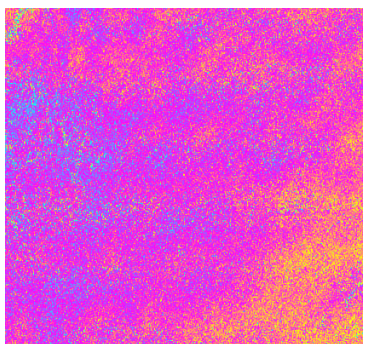

(c)

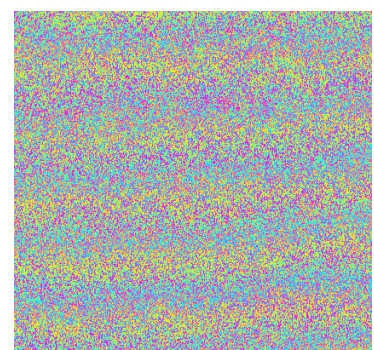

(d)

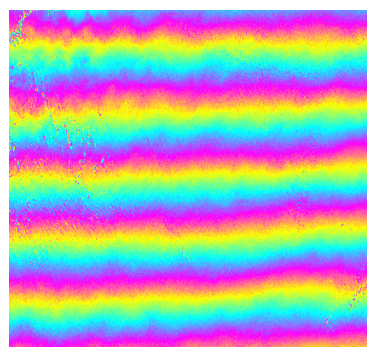

(e)

Fig. 3. The effect of azimuth coregistration error on the resulting interferogram in fullaperture processing. (a) is the azimuth profile of a target focused using full-aperture algorithm. The green envelope is the corresponding single burst focusing result. (b), (c), (d) and (e) represent different azimuth coregistration errors at locations shown in (a) and the corresponding interferograms. Data are from subswath 1 of an ALOS-2 ScanSAR scene acquired on 150402 . 


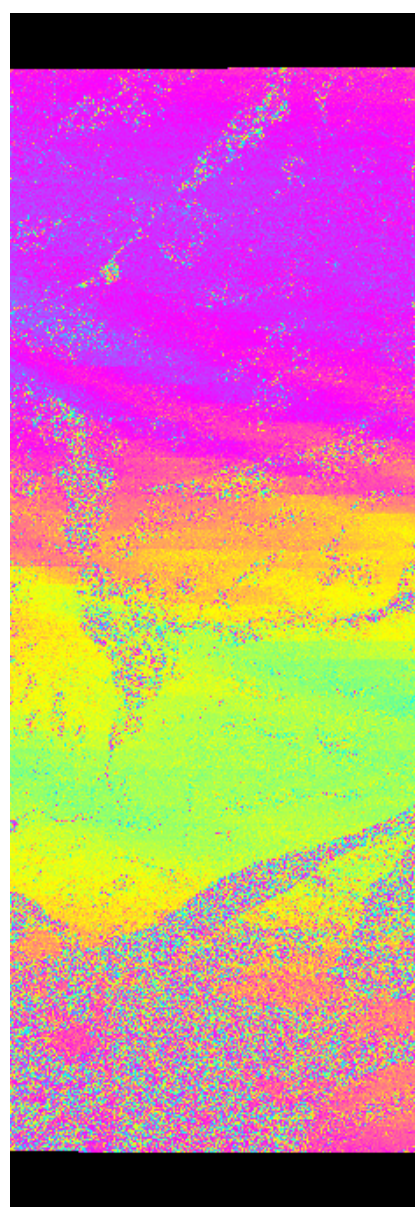

(a)
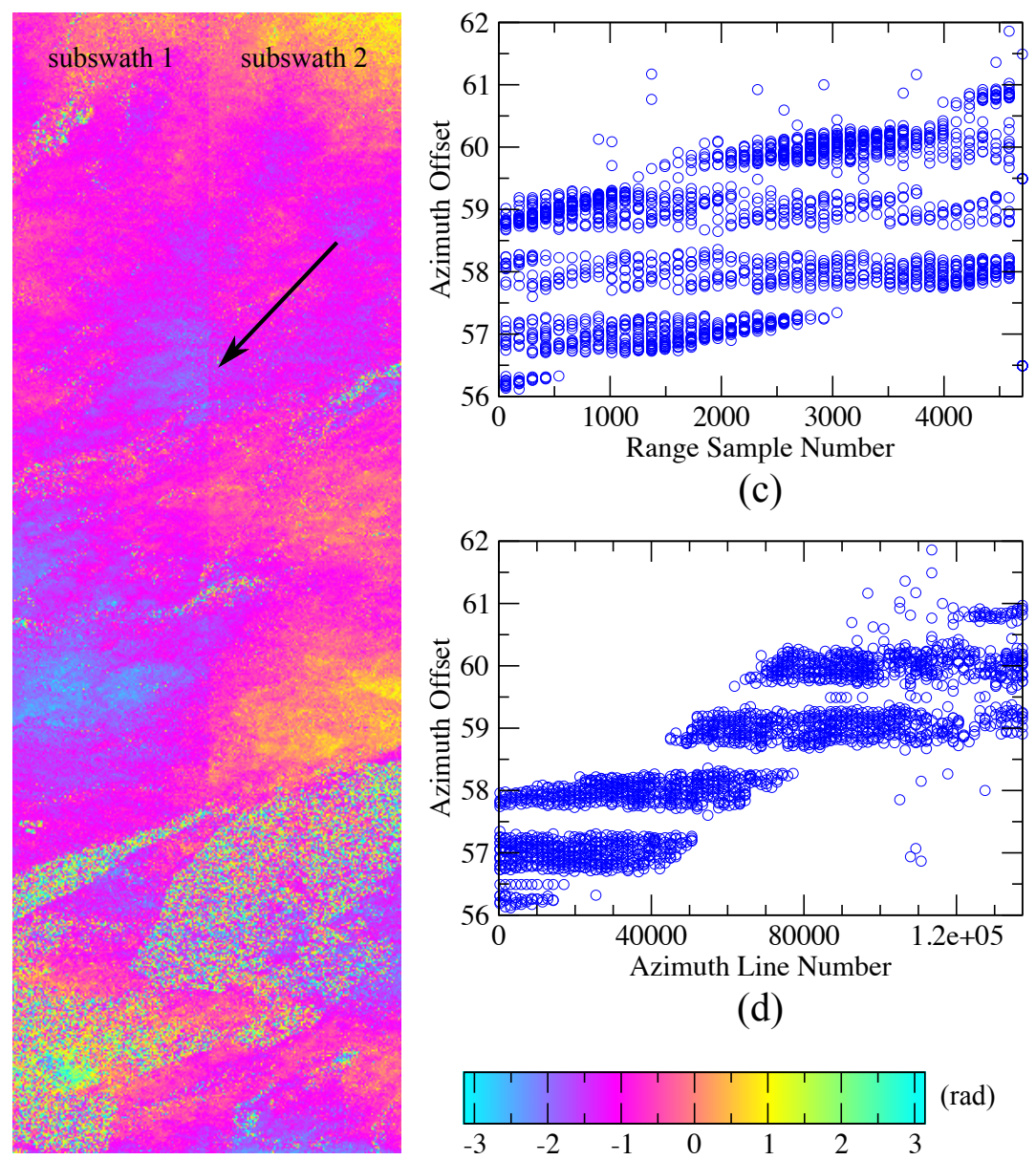

(c)

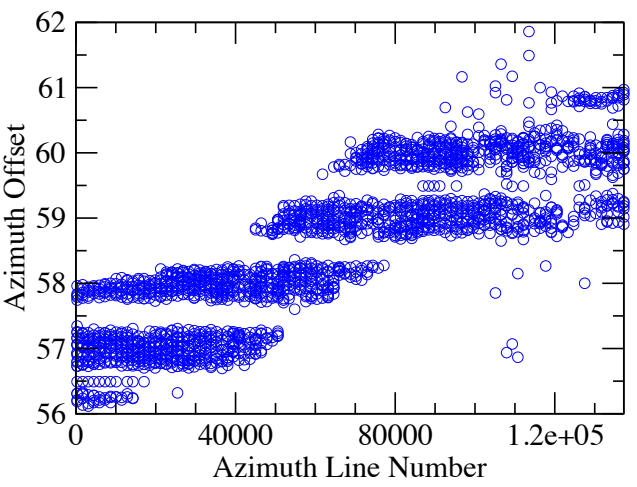

(d)

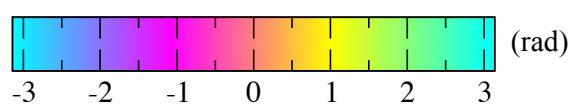

Fig. 4. Subswath phase inconsistency caused by coregistration error in the regular interferogram in full-aperture processing. (a) Spectral diversity interferogram of subswath 1 processed by burst-by-burst processing. (b) Phase inconsistency between subswath 1 and subswath 2 in the interferogram processed by full-aperture processing. Azimuth shift caused by the ionosphere seen from the azimuth offsets estimated by cross correlation shown as functions of range sample number (c) and azimuth line number (d). Data: 160331-161013. 


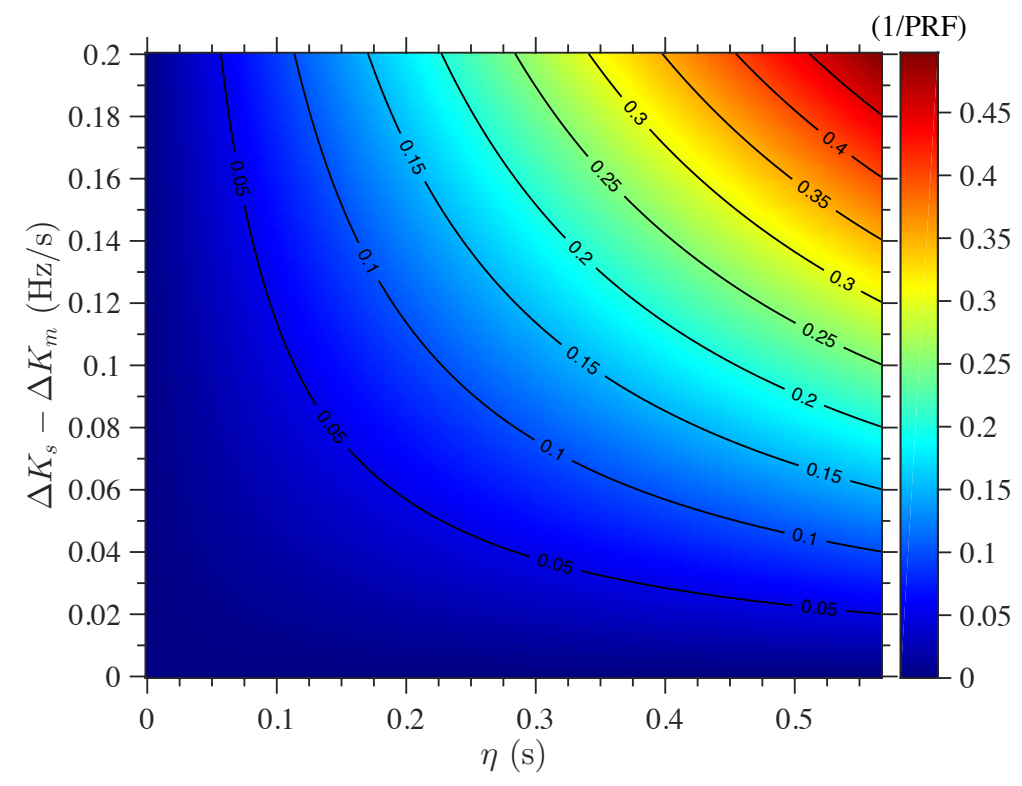

Fig. 5. Offset estimate error in spectral diversity coregistration caused by azimuth FM rate error as a function of the azimuth time (horizontal axis) and the azimuth FM rate error difference between the master and slave (vertical axis). Parameters are from subswath 1 of 150219 .

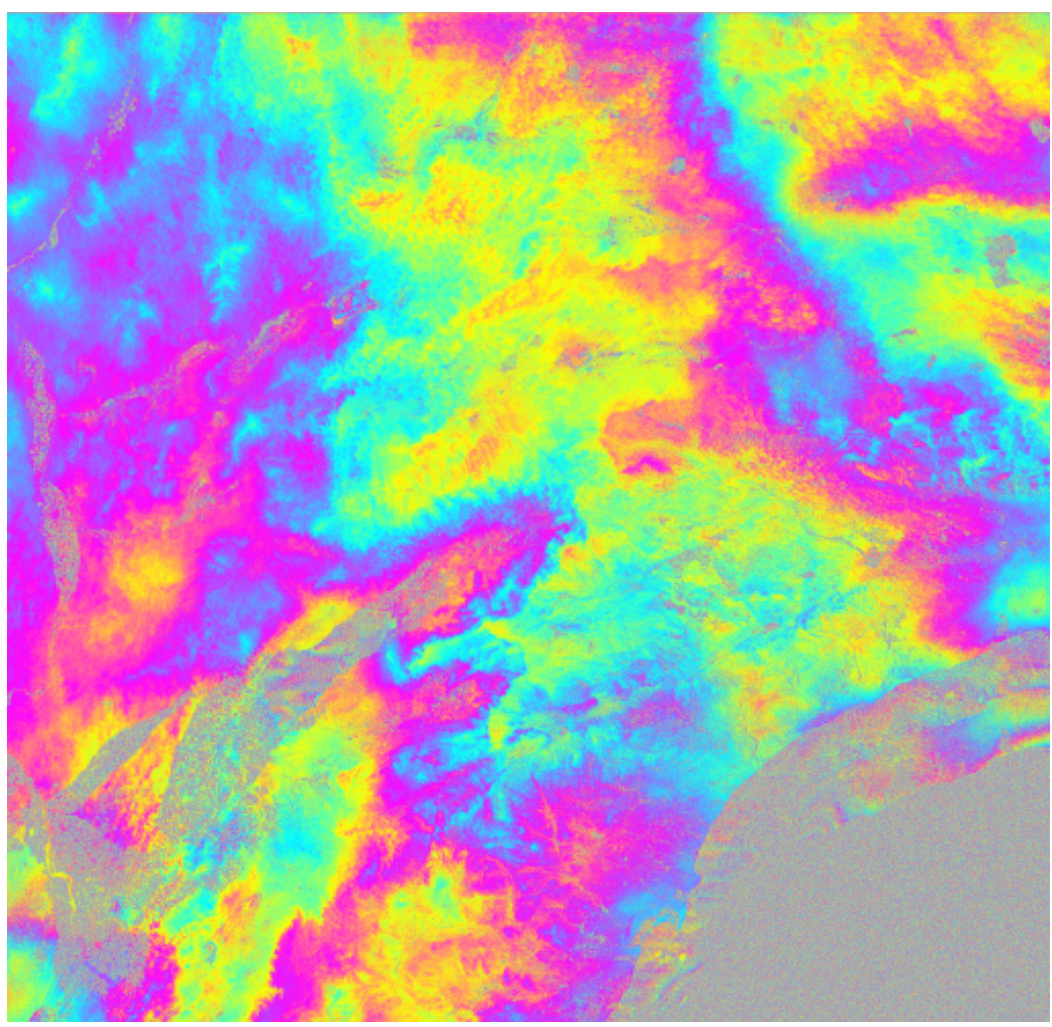


Fig. 6. The strong large-scale tropospheric phase in ALOS-2 ScanSAR interferogram after correction for ionospheric variations. InSAR pair: 161013-170720, wrapped at original $12.1 \mathrm{~cm}$ or $2 \pi$ radians.

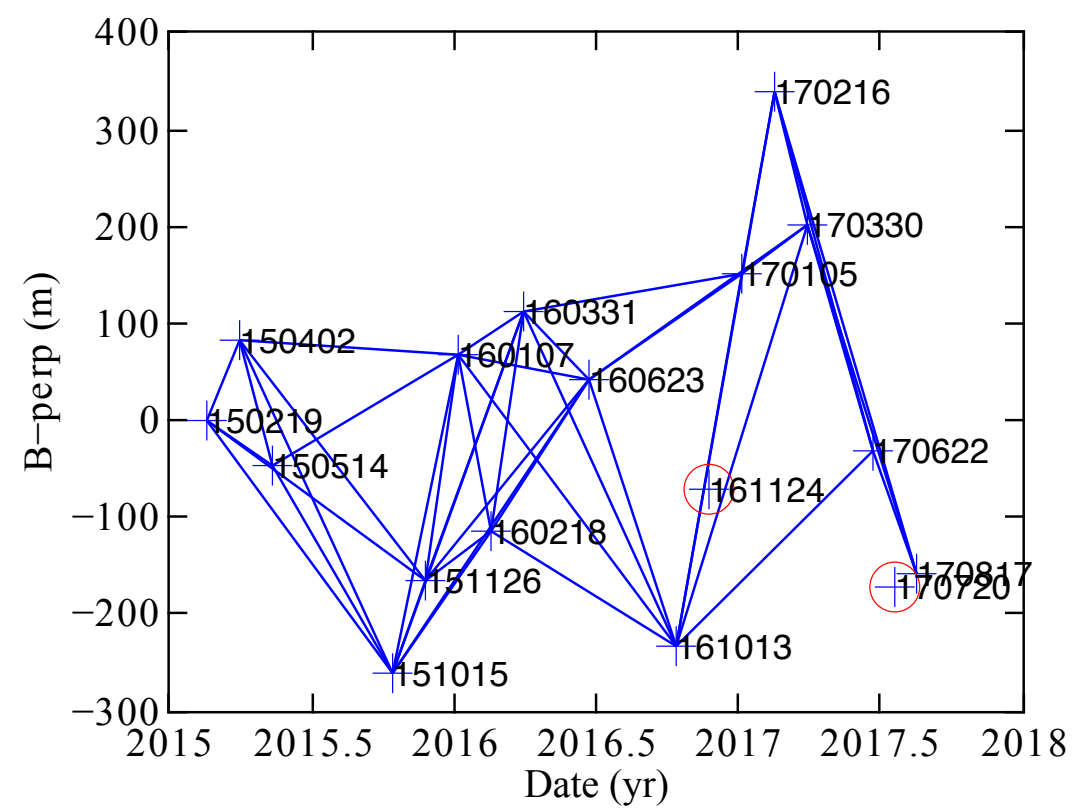

Fig. 7. The perpendicular baselines of the acquisitions used in this research. Blue lines show InSAR pairs that were calculated. The two acquisitions 161124 and 170720 were not used in the time series analysis because the tropospheric phase in the two acquisitions is very different. 


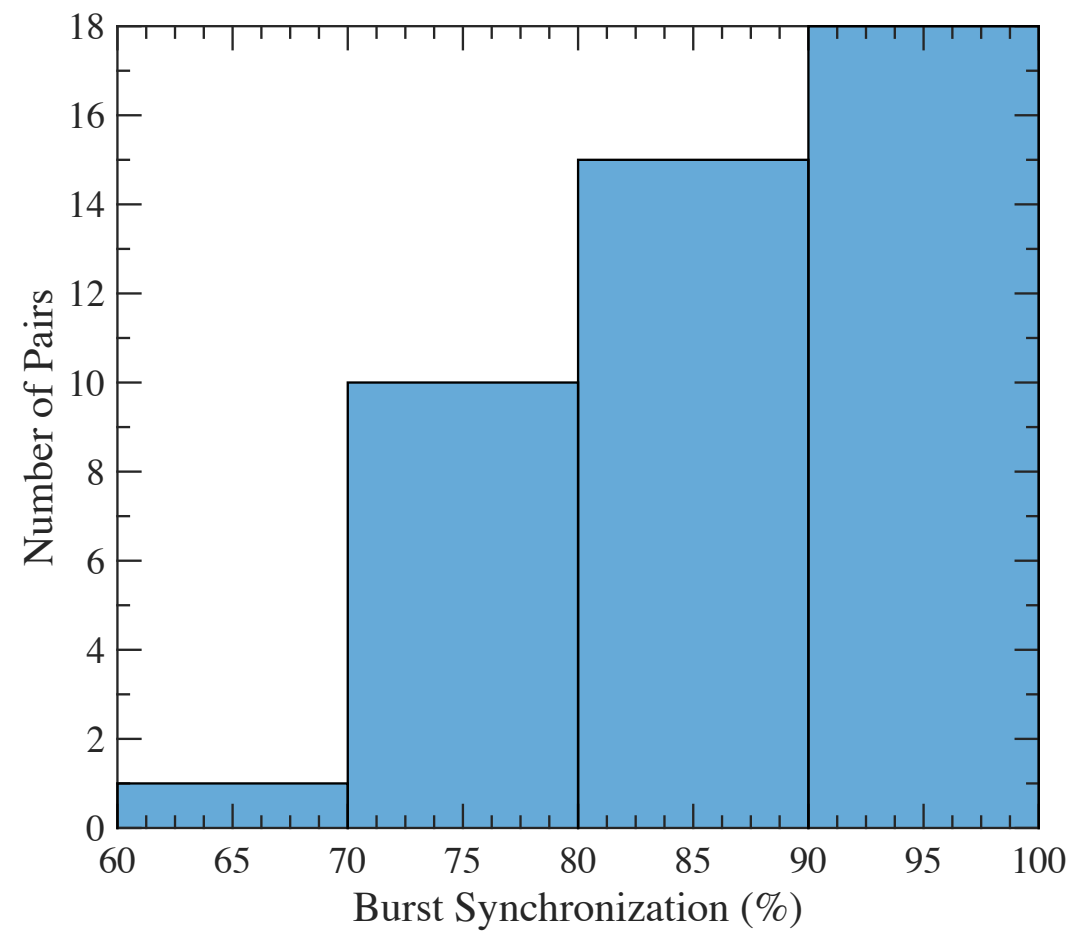

Fig. 8. The histogram of the burst overlaps for the InSAR pairs used in the time series analysis.
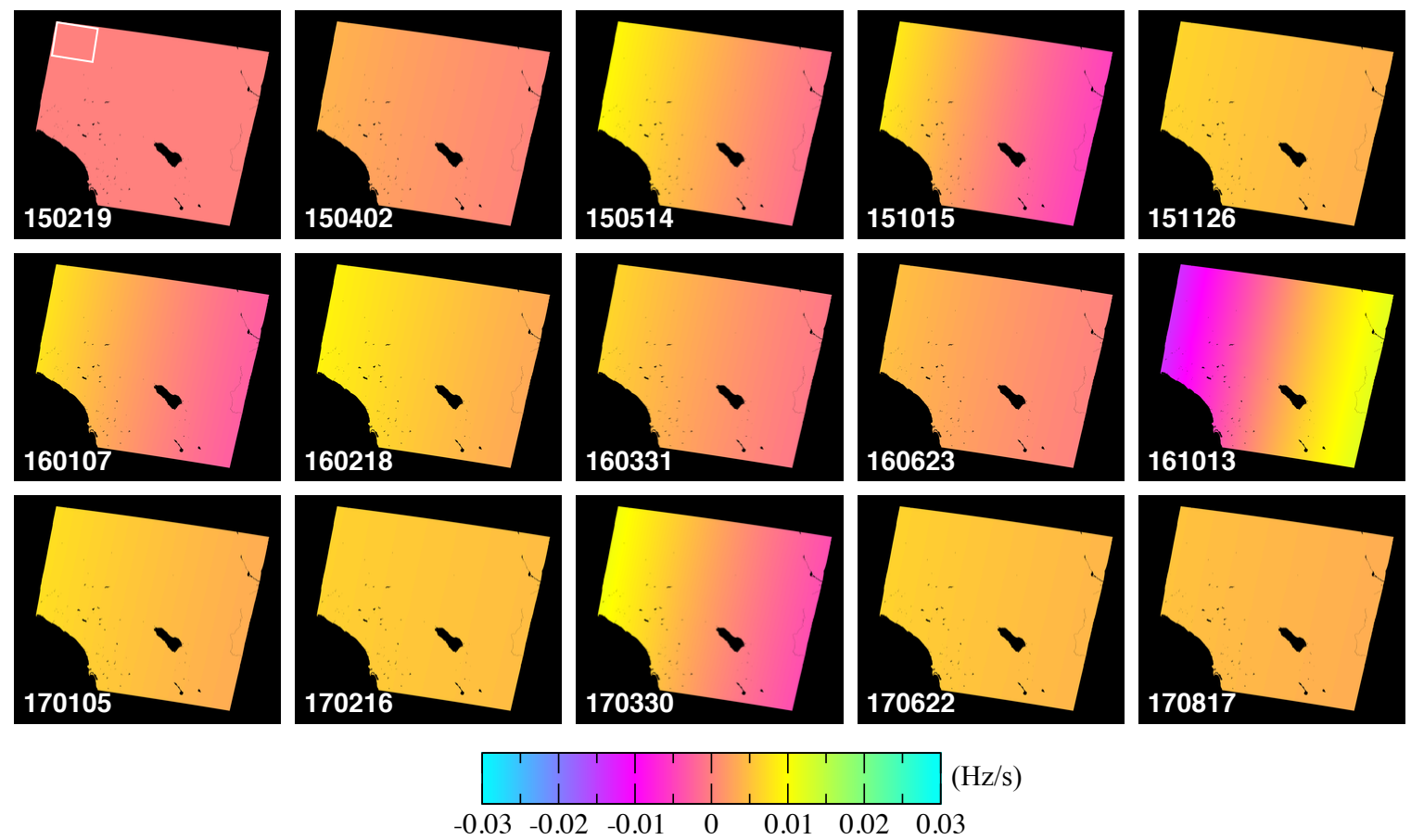

Fig. 9. The azimuth FM rate errors of the ALOS-2 products. The azimuth FM rate error of the first acquisition 150219 is assumed to be zero. The result of 161013 is biased due to the strong azimuth shift caused by the ionosphere which affects the estimation process. The small rectangle in 150219 shows the area covered by a stripmap acquisition. 

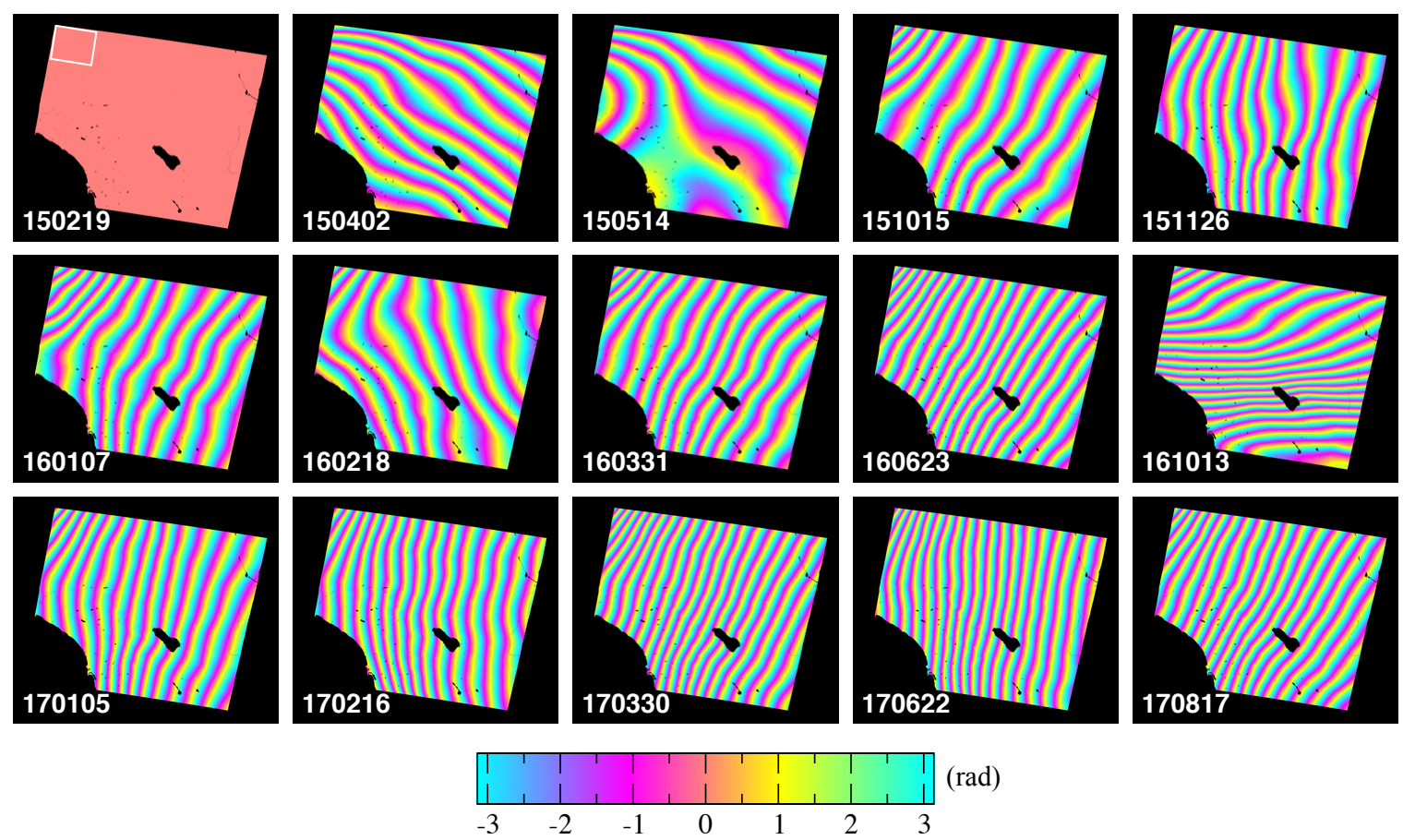

Fig. 10. The ionospheric phase of each ALOS-2 acquisition. The ionospheric phase of 150219 is assumed to be zero. The small rectangle in 150219 shows the area covered by a stripmap acquisition.
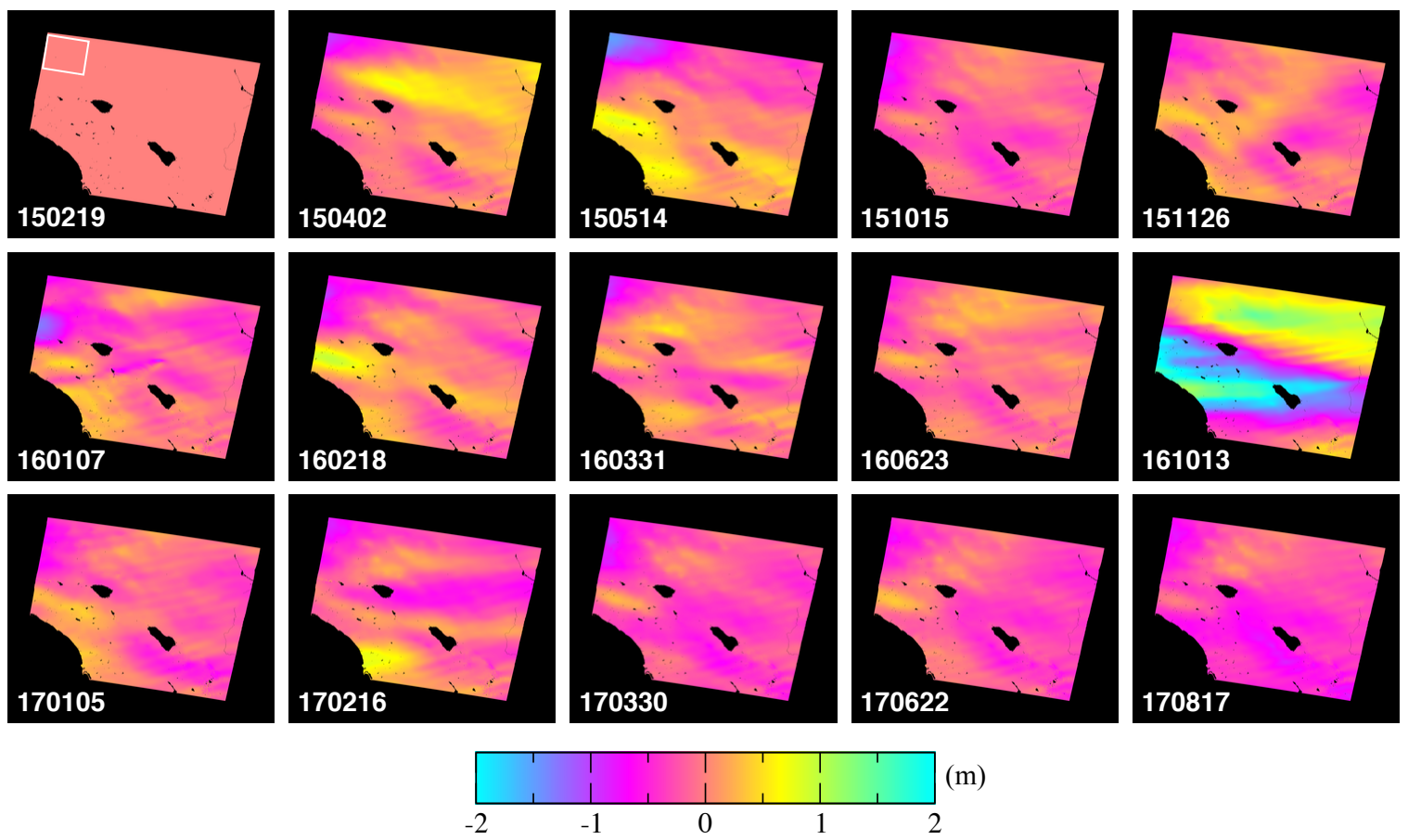

Fig. 11. The azimuth shift of each ALOS-2 acquisition caused by the ionosphere. The azimuth shift of 150219 is assumed to be zero. The small rectangle in 150219 shows the area covered by a stripmap acquisition. 

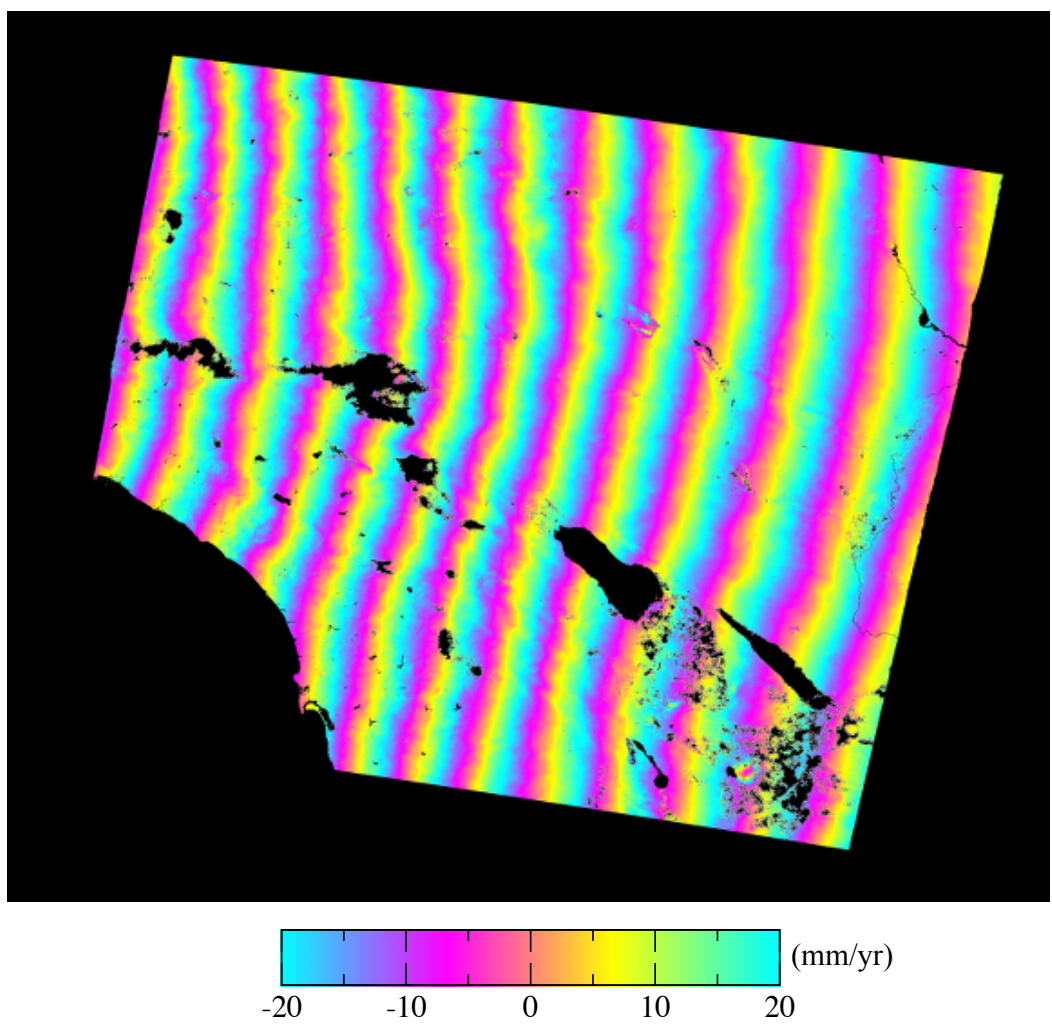

Fig. 12. Mean velocity map processed using full-aperture interferograms without ionospheric corrections. 


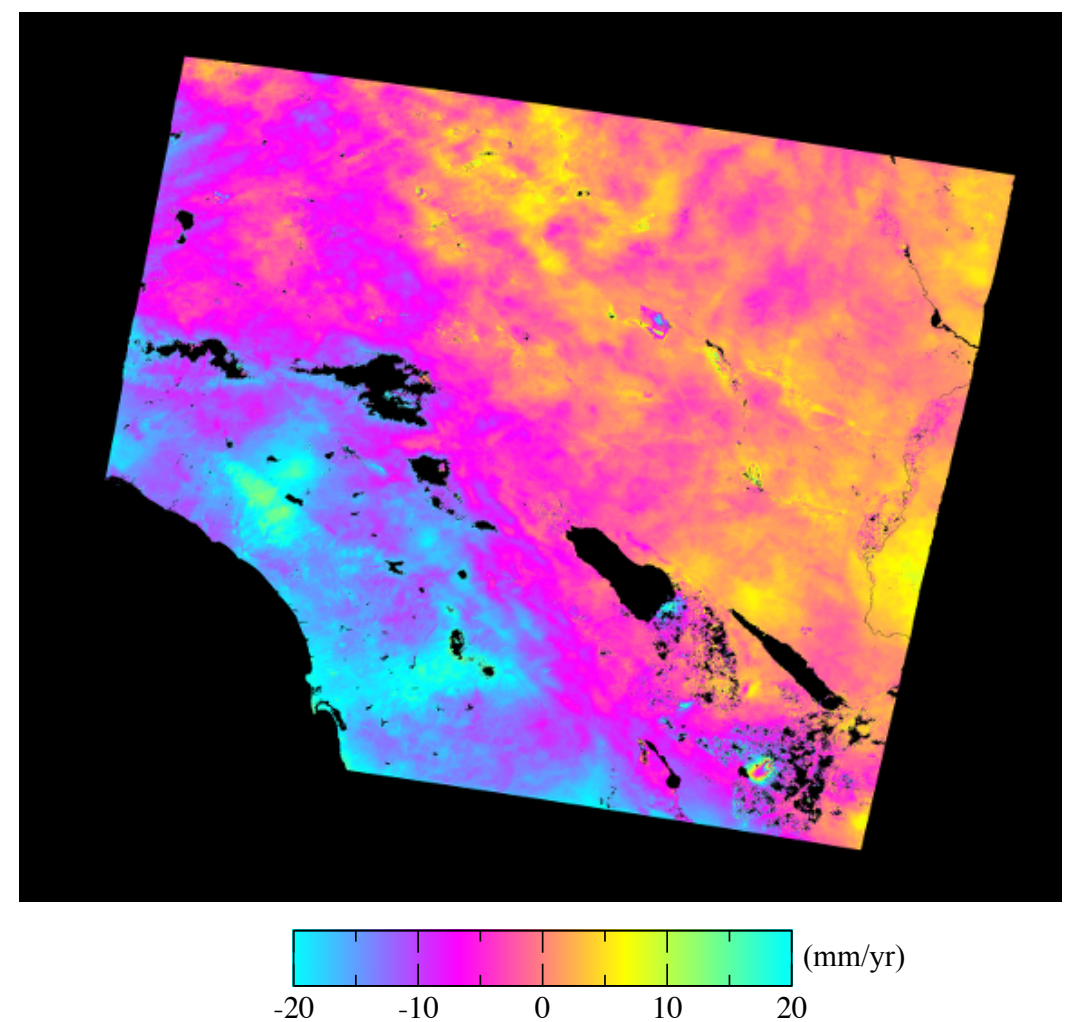

Fig. 13. Mean velocity map processed using full-aperture interferograms with ionospheric corrections.
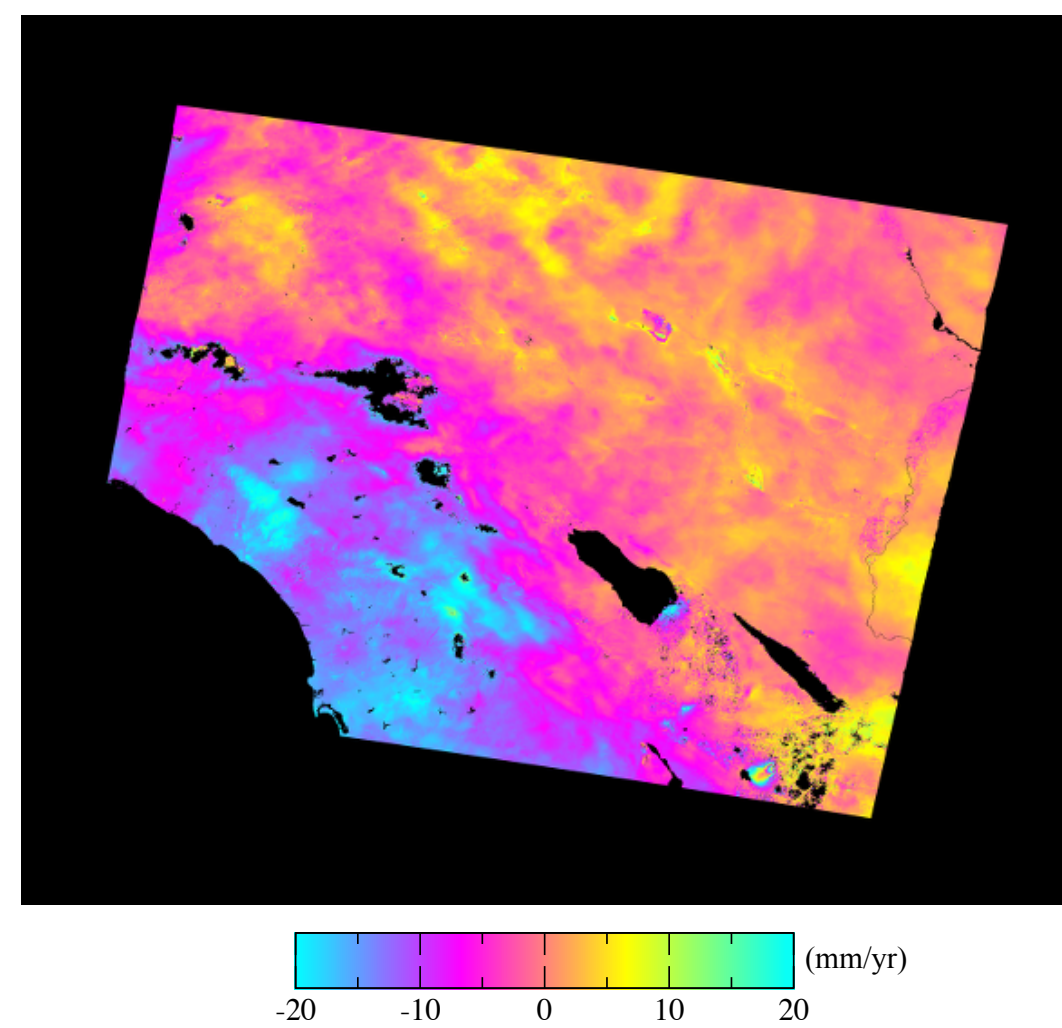
Fig. 14. Mean velocity map processed using burst-by-burst interferograms with ionospheric corrections.

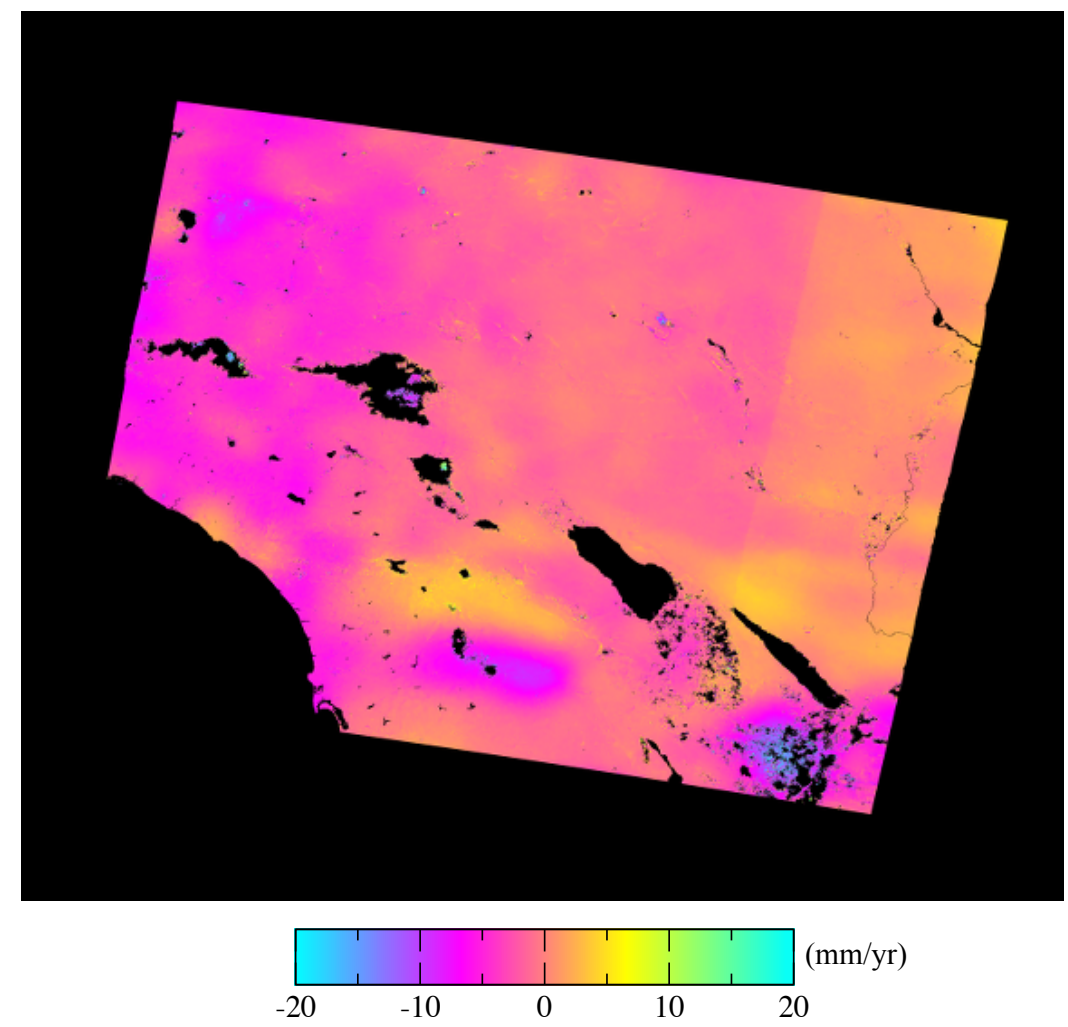

Fig. 15. Difference of mean velocity maps processed using full-aperture interferograms with ionospheric corrections and burst-by-burst interferograms with ionospheric corrections.

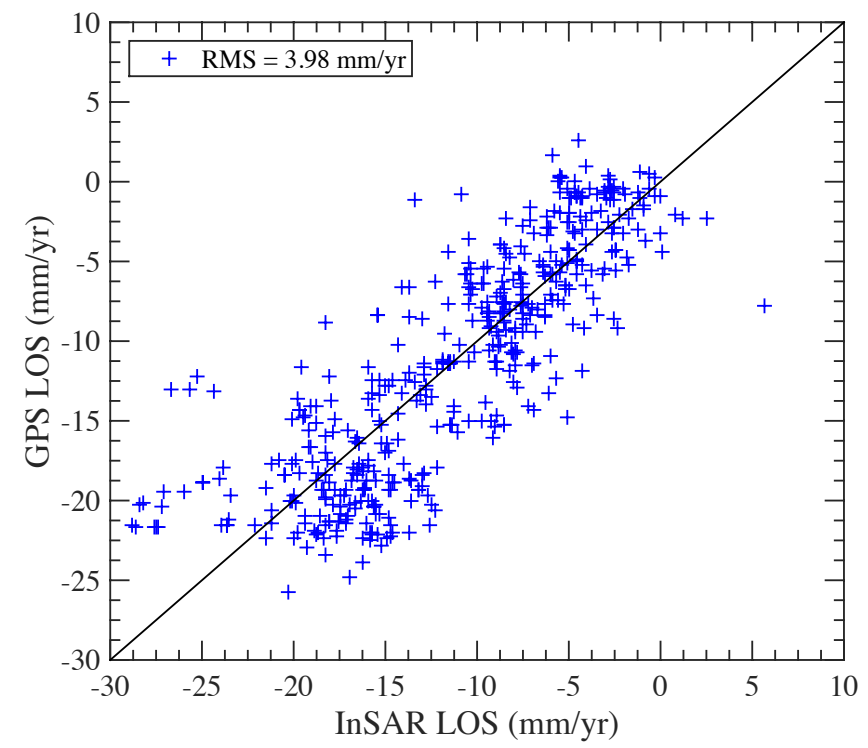


Fig. 16. Comparison of GPS velocities and InSAR velocities processed using fullaperture interferograms. The vertical GPS velocities are set to zero before projecting into SAR LOS directions.

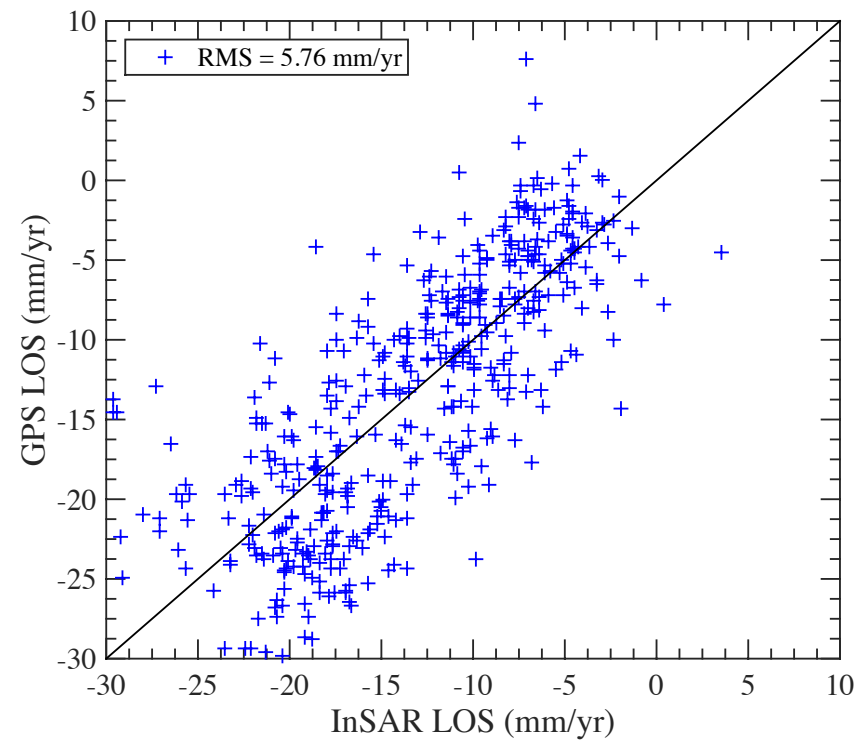

Fig. 17. Comparison of GPS velocities and InSAR velocities processed using fullaperture interferograms. The vertical GPS velocities are not set to zero before projecting into SAR LOS directions.

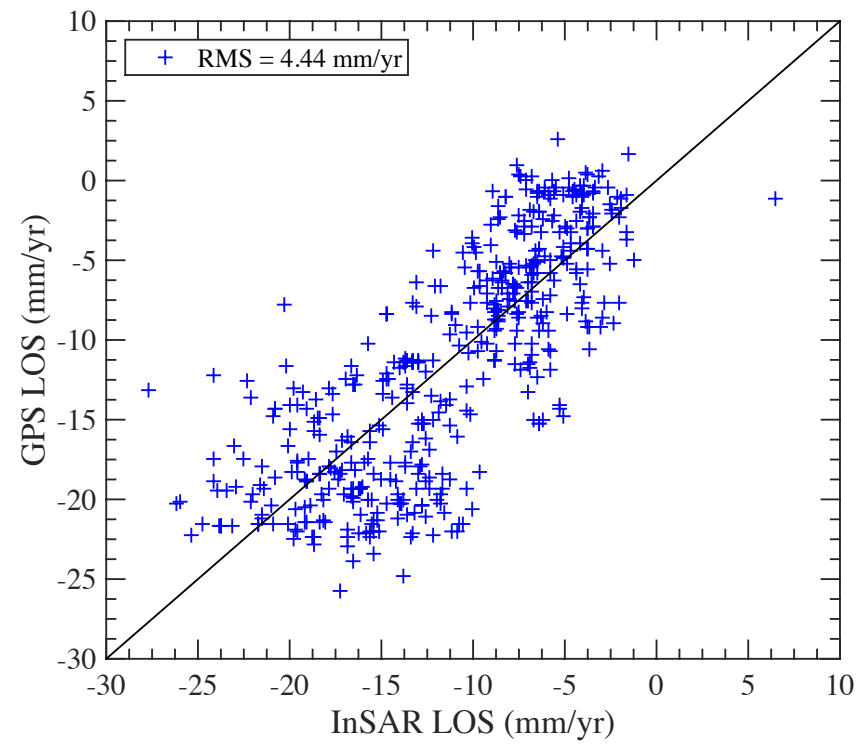

Fig. 18. Comparison of GPS velocities and InSAR velocities processed using burst-byburst interferograms. The vertical GPS velocities are set to zero before projecting into SAR LOS directions. 


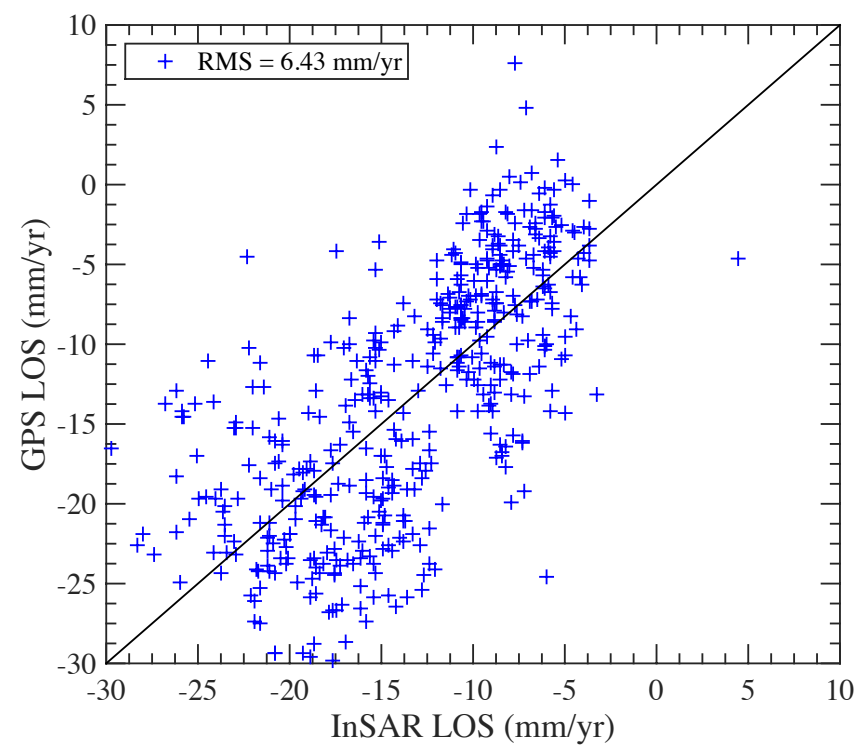

Fig. 19. Comparison of GPS velocities and InSAR velocities processed using burst-byburst interferograms. The vertical GPS velocities are not set to zero before projecting into SAR LOS directions. 


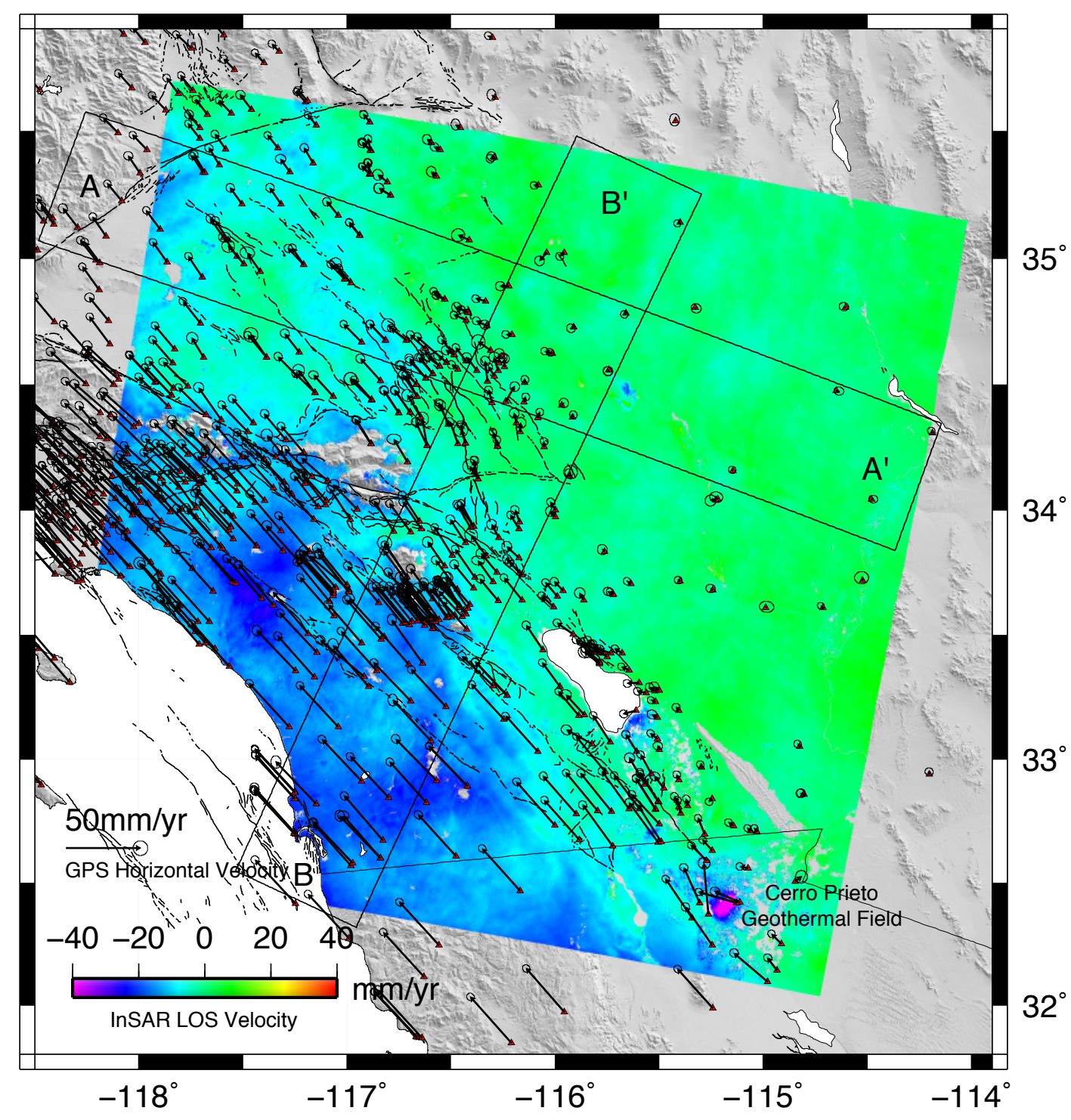

Fig. 20. InSAR LOS velocity map processed using full-aperture interferograms with ionospheric corrections (same as Fig. 13). GPS horizontal velocities are shown as black arrows with $95 \%$ confidence ellipses. The triangles indicate the GPS stations. The thin black lines are fault traces. $A A^{\prime}$ and $B B^{\prime}$ outline the two profiles used to compare InSAR and GPS velocities in Fig. 21 and Fig. 22. 


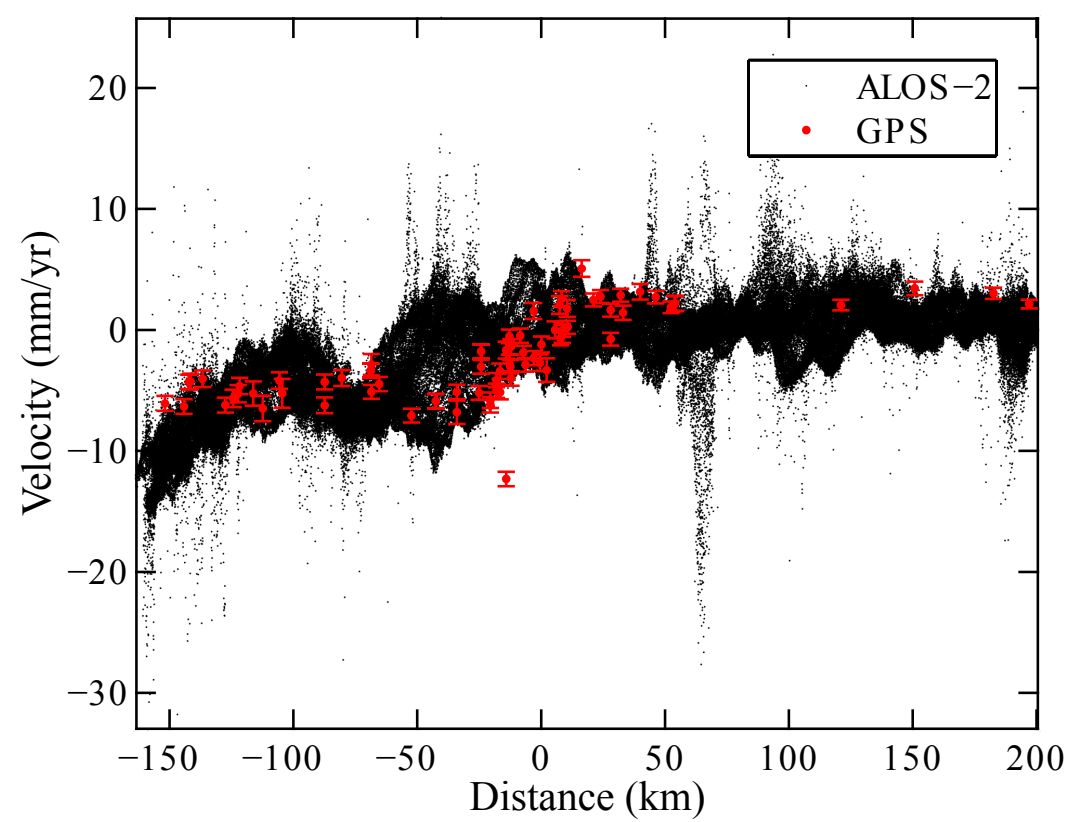

Fig. 21. InSAR and GPS velocities in SAR LOS direction along profile $A A^{\prime}$ shown in Fig. 20.

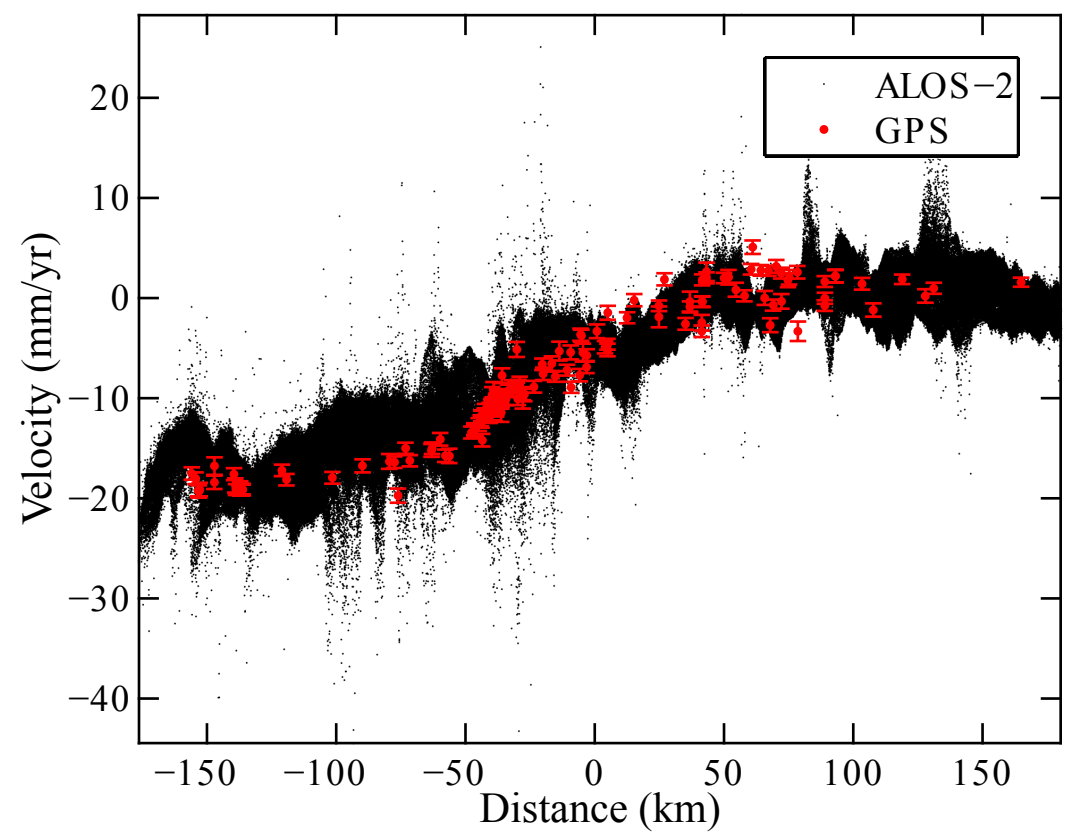

Fig. 22. InSAR and GPS velocities in SAR LOS direction along profile $B B^{\prime}$ shown in Fig. 20. 
Table I Parameters of the ALOS-2 default mode (from full-aperture product).

\begin{tabular}{|l|l|}
\hline Parameter & Value \\
\hline Operation Mode & ScanSAR / WD1 \\
\hline Beam Number & W2 \\
\hline Wavelength [cm] & 24.24525 \\
\hline Repeat Time [days] & 14 \\
\hline Number of Subswaths & $5(350 \mathrm{~km})$ \\
\hline Look Angle & $25.71^{\circ}-49.14^{\circ}$ \\
\hline Burst Lengths (s1-s5) [s] & $0.1345,0.1418,0.1488,0.1563,0.1726$ \\
\hline Burst Cycle Length [s] & 0.7838 \\
\hline Number of Looks of the ScanSAR System & $\sim 4$ \\
\hline Number of Complete Bursts in One Frame & $\sim 60$ (each subswath)* \\
\hline PRF / Azimuth Sampling Rate (s1-s5) [Hz] & $2661.847,3314.512,2406.568,2270.575,2821.225^{*}$ \\
\hline Azimuth Sample Size on Ground (s1-s5) [m] & $2.61,2.09,2.88,3.05,2.46^{*}$ \\
\hline Azimuth Resolution on Ground (s1-s5) [m] & $85.15,84.99,85.50,86.22,82.79$ \\
\hline Range Resampling Rate [MHz] & 17.47 \\
\hline Range Bandwidth [MHz] & 14 (nominal) / 11.90 (actual) \\
\hline Slant Range Sample Size [m] & 8.58 \\
\hline Slant Range Resolution [m] & 10.71 (nominal) / 12.60 (actual) \\
\hline
\end{tabular}

* parameters specific to full-aperture product.

Table II Parameters of the ALOS-2 and NISAR default modes. 


\begin{tabular}{|c|c|c|}
\hline Parameter & ALOS-2 & NISAR \\
\hline Launch Date & $\begin{array}{l}24 \text { May } 2014 \\
\text { (UTC) }\end{array}$ & $\begin{array}{c}2021 \\
\text { (expected) }\end{array}$ \\
\hline Mission Duration & $\begin{array}{l}5 \text { years } \\
\text { (goal is } 7 \text { years) }\end{array}$ & $\begin{array}{c}3 \text { years } \\
\text { (with extended mission fuel reserve) }\end{array}$ \\
\hline Altitude & $628 \mathrm{~km}$ & $\sim 740 \mathrm{~km}$ \\
\hline Repeat Time & $\begin{array}{c}14 \text { days } \\
\text { (acquires data every } \sim 2 \text { months) }\end{array}$ & $\begin{array}{l}12 \text { days } \\
\text { (expected to acquire data frequently) }\end{array}$ \\
\hline Wide Swath Mode & ScanSAR & SweepSAR \\
\hline Swath Width & $350 \mathrm{~km}$ & $240 \mathrm{~km}$ \\
\hline Look Angle & $\sim 26^{\circ}-49^{\circ}$ & $\sim 34^{\circ}-48^{\circ}$ \\
\hline Global Coverage & $\begin{array}{l}\text { ascending/descending tracks } \\
\text { (mostly descending tracks) }\end{array}$ & ascending/descending tracks \\
\hline Wavelength & $\begin{array}{c}\text { L-band } \\
(1236.5 \mathrm{MHz})\end{array}$ & $\begin{array}{l}\text { L-band } \\
(1260 \mathrm{MHz})\end{array}$ \\
\hline Range Bandwidth & $\begin{array}{c}14 \mathrm{MHz} \\
\text { (actual } 11.90 \mathrm{MHz} \text { ) }\end{array}$ & $20+5 \mathrm{MHz}$ \\
\hline Azimuth Resolution & $\begin{array}{l}\sim 85 \mathrm{~m} \\
(4 \text { looks })\end{array}$ & $\sim 7 \mathrm{~m}$ \\
\hline
\end{tabular}

